\title{
Tendon-derived cathepsin K-expressing progenitor cells activate Hedgehog signaling to drive heterotopic ossification
}

\author{
Heng Feng, ${ }^{1}$ Wenhui Xing, ${ }^{1}$ Yujiao Han, ${ }^{1}$ Jun Sun, ${ }^{1}$ Mingxiang Kong, ${ }^{2}$ Bo Gao,,${ }^{1,3}$ Yang Yang, ${ }^{1}$ Zi Yin, ${ }^{4}$ Xiao Chen, ${ }^{4}$ \\ Yun Zhao, ${ }^{1}$ Qing $\mathrm{Bi}^{2}{ }^{2}$ and Weiguo Zou ${ }^{1,5}$ \\ ${ }^{1}$ State Key Laboratory of Cell Biology, Shanghai Institute of Biochemistry and Cell Biology, CAS Center for Excellence in Molecular Cell Science, Chinese Academy of Sciences, University of Chinese Academy \\ of Sciences, Shanghai, China. ${ }^{2}$ Department of Orthopedics and Joint Surgery, Zhejiang Provincial People's Hospital, Hangzhou, China. ${ }^{3}$ Institute of Orthopedic Surgery, Xijing Hospital, Fourth Military Medical \\ University, Xi'an, China. ${ }^{4}$ Key Laboratory of Tissue Engineering and Regenerative Medicine of Zhejiang Province, School of Medicine, Zhejiang University, Hangzhou, China. ${ }^{5}$ Institute of Microsurgery on \\ Extremities, Shanghai Jiao Tong University Affiliated Sixth People's Hospital, Shanghai, China.
}

\begin{abstract}
Heterotopic ossification ( $\mathrm{HO}$ ) is pathological bone formation characterized by ossification within muscle, tendons, or other soft tissues. However, the cells of origin and mechanisms involved in the pathogenesis of $\mathrm{HO}$ remain elusive. Here we show that deletion of suppressor of fused (Sufu) in cathepsin K-Cre-expressing (Ctsk-Cre-expressing) cells resulted in spontaneous and progressive ligament, tendon, and periarticular ossification. Lineage tracing studies and cell functional analysis demonstrated that Ctsk-Cre could label a subpopulation of tendon-derived progenitor cells (TDPCs) marked by the tendon marker Scleraxis (Scx). Ctsk ${ }^{+} S c x^{+}$TDPCs are enriched for tendon stem cell markers and show the highest self-renewal capacity and differentiation potential. Sufu deficiency caused enhanced chondrogenic and osteogenic differentiation of Ctsk-Cre-expressing tendon-derived cells via upregulation of Hedgehog ( $\mathrm{Hh})$ signaling. Furthermore, pharmacological intervention in Hh signaling using JQ1 suppressed the development of HO. Thus, our results show that Ctsk-Cre labels a subpopulation of TDPCs contributing to HO and that their cell-fate changes are driven by activation of Hh signaling.
\end{abstract}

\section{Introduction}

Heterotopic ossification ( $\mathrm{HO}$ ) is a pathological process in which ectopic bone forms within muscles, tendons, or other soft tissues (1). HO currently develops after trauma induced by deep burns, fractures, total hip arthroplasty, and so on $(2,3)$. HO is also a typical symptom of some rare genetic diseases, such as fibrodysplasia ossificans progressiva and progressive osseous heteroplasia $(\mathrm{POH})(4) . \mathrm{HO}$ often affects tendons and ligaments containing progenitor cells. To date, various types of progenitors, including tendon-derived progenitor cells (TDPCs), circulating progenitor cells, and progenitor cells from peripheral nerves, have been identified as the origin of HO (5-12). TDPCs have been well studied in vitro. Bi et al. identified and characterized a unique cell population of tendon stem/progenitor cells expressing Sca1, CD90.2, and CD44 (13). Additionally, bone-cartilage-stromal progenitors characterized by $\mathrm{Tie}^{-} \mathrm{CD} 45^{-} \mathrm{Thy}^{-} 6 \mathrm{C}^{-}{ }^{-} \alpha_{\mathrm{v}}{ }^{+} \mathrm{CD} 105^{+}$contribute to HO (14). Previous studies have shown that TDPCs characterized by the tendon marker Scleraxis $(S c x)$ contribute to the formation of

Authorship note: HF, WX, and YH contributed equally to this work. Conflict of interest: The authors have declared that no conflict of interest exists. Copyright: (c) 2020, American Society for Clinical Investigation.

Submitted: August 16, 2019; Accepted: August 20, 2020; Published: November 3, 2020. Reference information: J Clin Invest. 2020;130(12):6354-6365.

https://doi.org/10.1172/JCl132518. chondrocytes and osteoblasts in $\mathrm{HO}(5,6)$. However, in vivo studies of TDPCs contributing to $\mathrm{HO}$ and the regulatory mechanisms involved in $\mathrm{HO}$ are limited.

Hedgehog $(\mathrm{Hh})$ signaling plays critical roles in cartilage and bone development, and the dysregulation of $\mathrm{Hh}$ signaling contributes to many bone diseases, such as bone tumors, osteoarthritis, and HO (15-19). Suppressor of fused (Sufu), the major negative regulator of Hh signaling, sequesters full-length Gli proteins in the cytoplasm to restrict their activity, and inactivation of Sufu leads to severe ectopic Hh pathway activation $(20,21)$. The $\mathrm{HO}$ in $\mathrm{POH}$ suggests that elevated basal $\mathrm{Hh}$ signaling is sufficient to induce ectopic osteogenic differentiation of the soft tissues through intramembranous ossification (19). Studies have demonstrated the participation of Hh signaling in $\mathrm{HO}$ by endochondral ossification, but have not shown the cell-autonomous effects of dysregulated Hh signaling or identified the cell population that responds to Hh signaling.

Here, we identified a subpopulation of TDPCs labeled by cathepsin $\mathrm{K}-\mathrm{Cre}$ (Ctsk-Cre) that contribute to HO. Ctsk has been proven to be a marker of mature osteoclasts, perichondrial progenitors in the perichondrial groove, and periosteum stem/progenitor cells (22-25). We found that upregulation of Hh signaling by inactivation of Sufu in Ctsk-Cre-expressing cells induced progressive $\mathrm{HO}$ in tendons and ligaments. Lineage tracing studies showed that Ctsk-Cre could label a subpopulation of TDPCs expressing the tendon marker $S c x$ in the midsubstance of the tendon that exhibit 
A

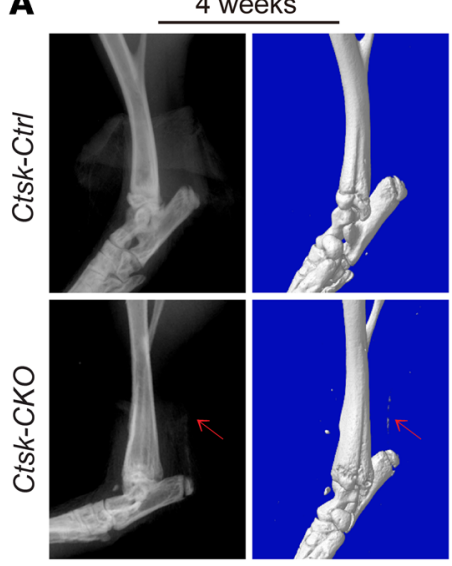

B

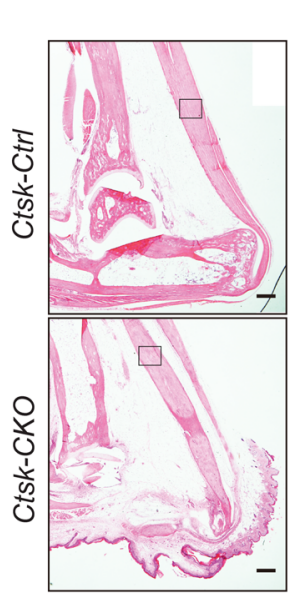

9 weeks

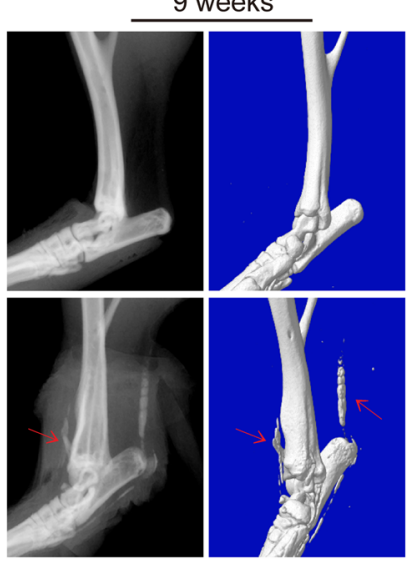

20 weeks

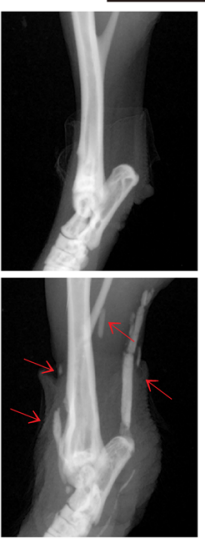

20 weeks
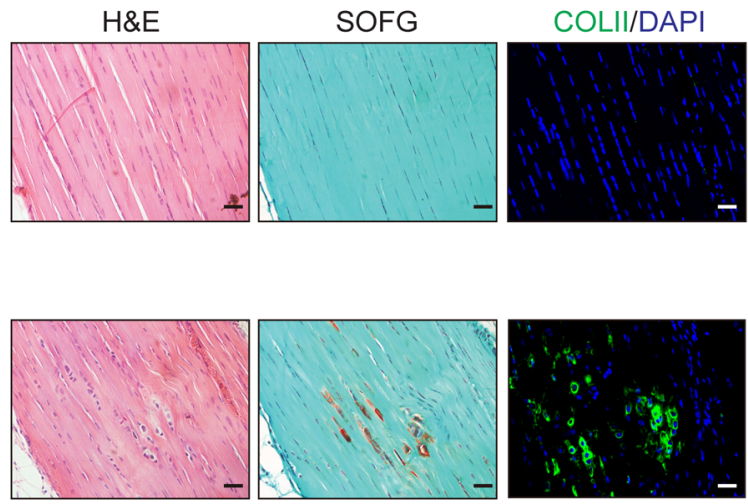

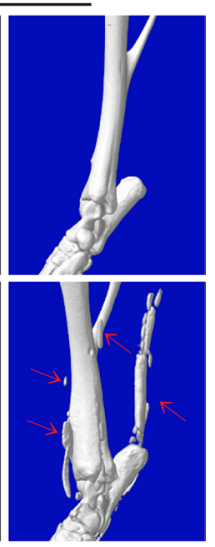

Figure 1. Sufu deletion in Ctsk-Creexpressing cells induces spontaneous $\mathrm{HO}$ in the ligament and tendon. (A) X-ray images and $\mu \mathrm{CT}$ scans reveal that spontaneous ossification (red arrows) of the tibiotalar ligament and Achilles tendon progresses gradually in Ctsk-CKO mice at 4, 9, and 20 weeks. Images are representative of 3 different sets of experiments. $n=3$ per group. (B) H\&E staining, Safranin $\mathrm{O} /$ fast green (SOFG) staining, and immunofluorescence staining of the chondrogenic marker type II collagen (COLII) and the osteogenic marker osteopontin (OPN) of the Achilles tendon from 20-week-old Ctsk-Ctrl and Ctsk-CKO mice. Images are representative of 3 different sets of experiments. Scale bars: $100 \mu \mathrm{m}(2$ left panels); $20 \mu \mathrm{m}$ (8 right panels).
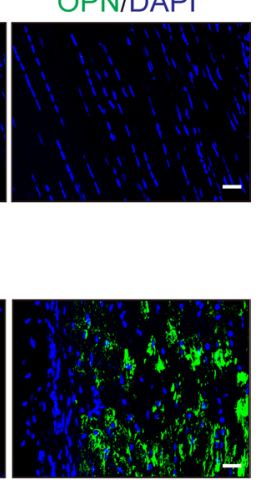

a strong self-renewal capacity and differentiation potential. Furthermore, pharmacological intervention in Hh signaling using the Hh signaling inhibitor JQ1 suppressed the development of HO. Overall, our results identified a subpopulation of TDPCs labeled by Ctsk-Cre, and demonstrated that activation of Hh signaling drives $\mathrm{HO}$ in tendons and ligaments in a cell-autonomous manner.

\section{Results}

Sufu deletion in Ctsk-Cre-expressing cells induces spontaneous ligament, tendon, and joint HO. A previous study reported that Ptpn11 deletion in Ctsk-Cre-expressing cells resulted in metachondromatosis, which is believed to be associated with overactive Hh signaling (25). To identify the mechanism of Hh signaling in cartilage tumors, we crossed Sufulflf mice with mice expressing Cre under the control of the endogenous Ctsk promoter (Supplemental Figure 1A; supplemental material available online with this article; https://doi.org/10.1172/ JCI132518DS1). From 4 weeks, the Ctsk-Cre Sufut/fll (hereafter Ctsk$\mathrm{CKO}$ ) mice began to show mobility problems. Surprisingly, $\mathrm{x}-\mathrm{ray} / \mu \mathrm{CT}$ (micro-CT) radiographs of the 4-, 9-, and 20-week-old Ctsk-CKO mice showed spontaneous and progressive periarticular, ligament, and tendon ossification, which was distinguished from the phenotype of the Ctsk-Cre Ptpn11//lf mice mimicking human metachondromatosis (Figure 1A and Supplemental Figure 1C). However, the Ctsk-Cre Sufut ${ }^{f /+}$ mice (hereafter Ctsk-Ctrl) showed normal bone characteristics (Supplemental Figure 1B). Ectopic ossification was detected in the ligaments and tendons of the hind paws in the 20-week-old Ctsk-CKO mice by $\mu \mathrm{CT}$ analysis (Supplemental Figure 1E). Moreover, ossified lesions were found in the ligaments and tendons of the forepaws and radius in the 40-week-old Ctsk-CKO mice (Supplemental Figure 1F). Histologically, Safranin O/fast green (SOFG) staining showed ectopic ossification at the patellar tendon (Supplemental Figure 1D, a). SOFG staining and immunofluorescence staining of type II collagen (COLII) showed chondrocytes in the midsubstance of the Achilles tendon from the 20-week-old Ctsk-CKO mice (Figure 1B). In addition, $\mathrm{H} \& \mathrm{E}$ staining and osteopontin (OPN) staining showed ectopic ossification at the Achilles tendon (Figure 1B). Furthermore, SOFG staining showed that osteochondroma, which is formed in the proximal tibia and femur, featured a cartilaginous cap and a marrow cavity with $100 \%$ penetrance (Supplemental Figure 1D, b). Interestingly, unlike the phenotype of Ctsk-Cre Ptpn11 $1 /$ /fl mice, enchondroma, which is a benign tumor that forms in the central part of the bone where bone marrow is stored, was not observed in the Ctsk-CKO mice (25). These results suggest that Sufu deletion in Ctsk-Cre-expressing cells induces spontaneous periarticular, ligament, and tendon ossification and osteochondromas.

Ctsk-Cre-expressing cells in the tendons and ligaments contribute to HO. To identify the cell origin for HO in the Ctsk-CKO mice, we performed lineage tracing studies using Ai9 reporter mice to label all Ctsk-lineage cells with tdTomato fluorescence (Figure 2A). Notably, Ctsk-Cre was expressed in the majority of cells within the 
A

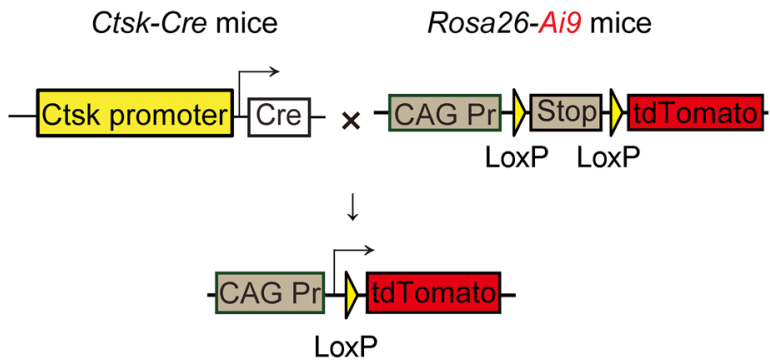

B

Achilles tendon Patellar tendon Quadriceps tendon
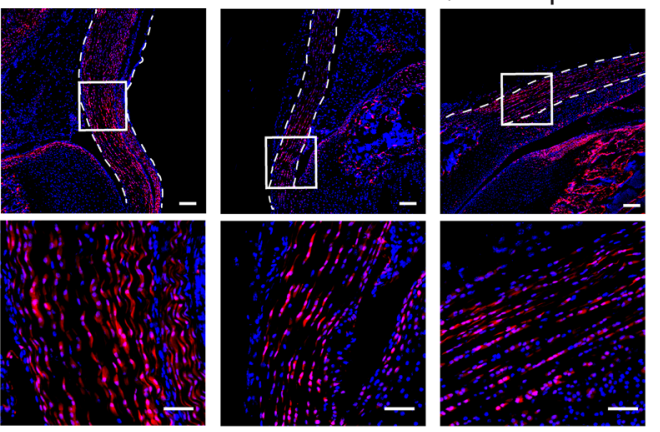

C

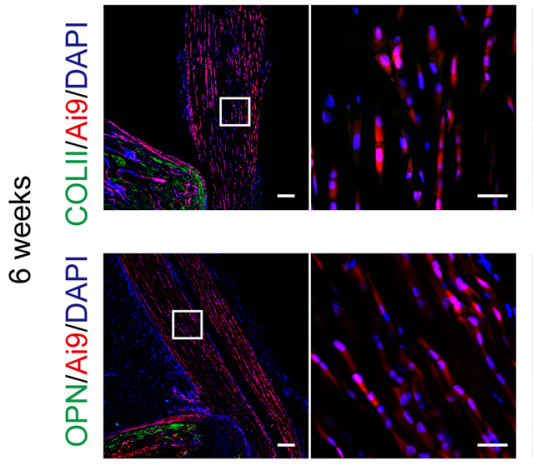

Ctsk-CKO Ai9
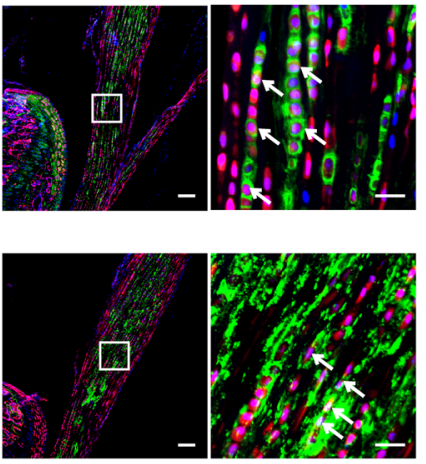

D $\stackrel{+}{\stackrel{+}{*}} 30-\quad$ *** $\quad \square$ Ctsk-Ctrl Aig

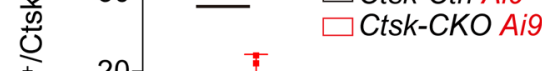

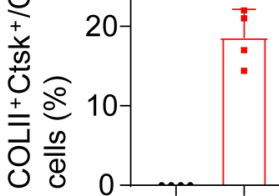

E

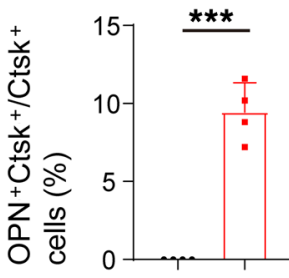

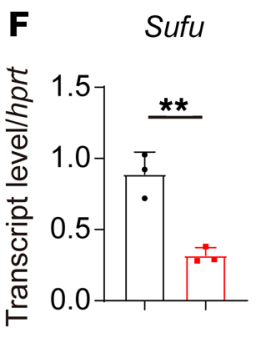

H Osx
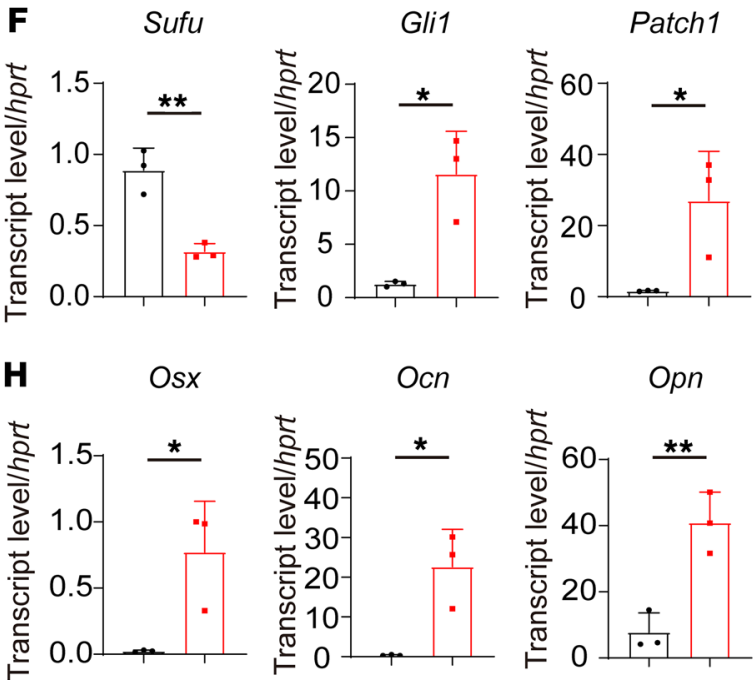

Opn
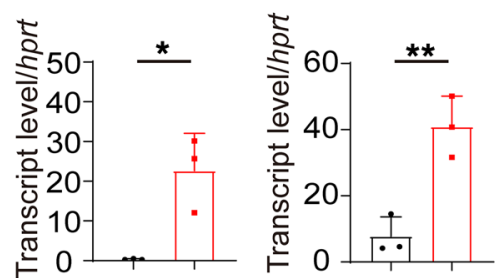

J

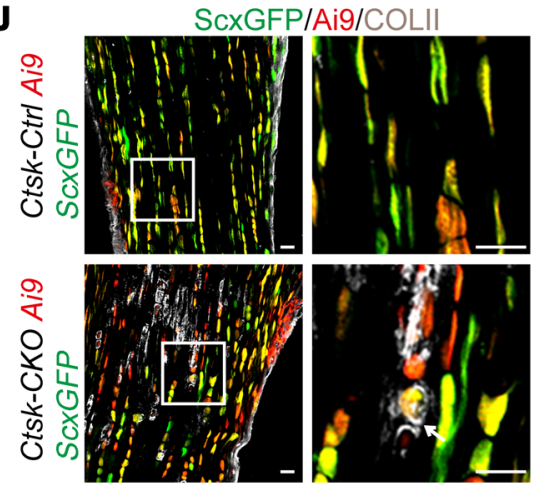

G $\quad \operatorname{sox} 9$
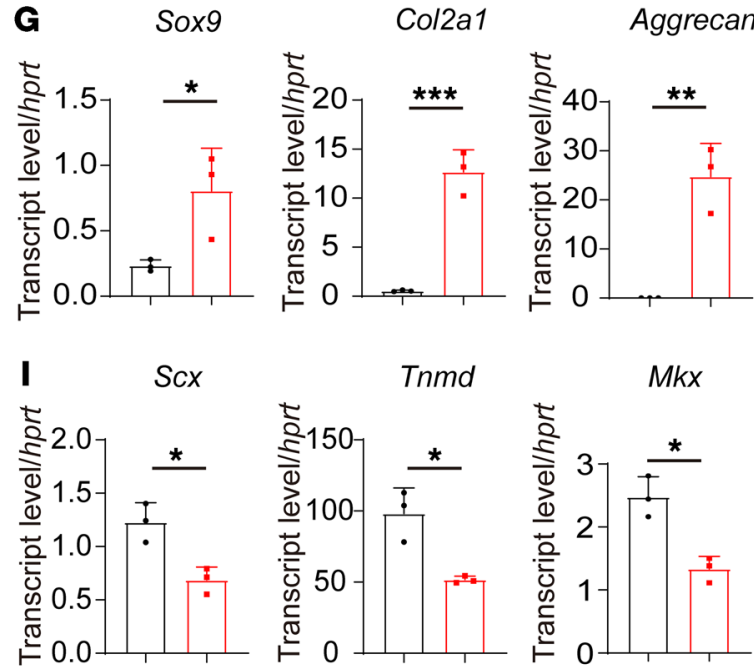

Aggrecan

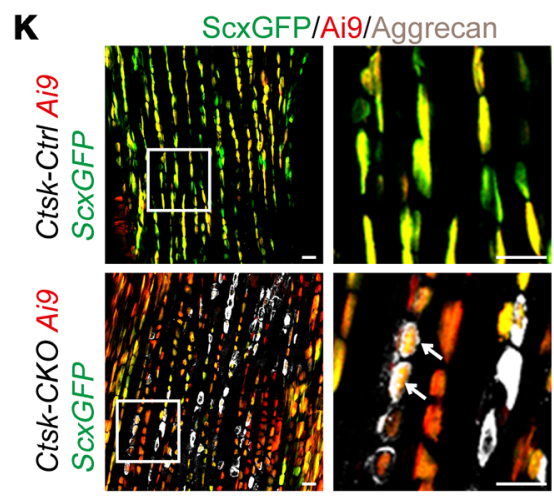


Figure 2. Ctsk-Cre-expressing cells in tendons and ligaments contribute to HO. (A) Schematic of the Ctsk-Cre Rosa26-Aig reporter mice. (B) Confocal images of hind limbs from Ctsk-Cre Rosa26-Aig mice showing Ctsk expression in the cells of the Achilles tendon, patellar tendon, and quadriceps tendon. Scale bars: $50 \mu \mathrm{m}$. (C) Immunofluorescence showing Ctsk ${ }^{+}$ (Ai9+) cells in the Achilles tendon of 6-week-old Ctsk-CKO Rosa26-Ai9 mice displays the chondrogenic marker COLII (green) and the osteogenic marker OPN (green). Scale bars: $50 \mu \mathrm{m}$ (left panels); $25 \mu \mathrm{m}$ (right panels). (D) Quantitative analysis of the percentage of $\mathrm{Ctsk}^{+}\left(\mathrm{Aig}^{+}\right)$cells that express the chondrogenic marker COLII in Achilles tendons. $n=4$ per group. (E) Quantitative analysis of the percentage of $\mathrm{Ctsk}^{+}\left(\mathrm{Aig}^{+}\right)$cells that express the osteogenic marker OPN in Achilles tendons. $n=4$ per group. $(\mathbf{F}-\mathbf{I})$ qRT-PCR analysis of Hh target gene $(\mathbf{F})$, chondrogenic gene (G), osteogenic gene $(\mathbf{H})$, and tendon-related gene (I) expression in sorted Ctsk ${ }^{+}$Achilles tendon cells from 6-week-old Ctsk-CKO Rosa26-Aig mice compared with Ctsk-Ctrl Rosa26-Ai9 mice. $n=3$ per group. (J and $\mathbf{K}$ ) Immunofluorescence showing $\mathrm{Ctsk}^{+} \mathrm{S} \mathrm{Cx}^{+}$(orange) cells in the Achilles tendon of 6-week-old CtskCKO Rosa26-Aig ScxGFP mice displays the chondrogenic marker COLII and aggrecan (gray). Scale bars: $20 \mu \mathrm{m}$. Results are presented as the mean \pm SEM; unpaired $t$ test, ${ }^{*} P<0.05,{ }^{* *} P<0.01,{ }^{* *} P<0.001$.

Achilles tendon, quadriceps tendon, and tendinous insertions of the patella (Figure 2B). We found increased expression levels of the chondrogenic marker COLII and the osteogenic marker OPN in the $\mathrm{Ctsk}^{+}\left(\mathrm{Ai}^{+}\right)$cells from the Achilles tendons of the 6-weekold Ctsk-CKO Rosa26-Ai9 mice compared with those in the CtskCtrl Rosa26-Ai9 mice (Figure 2, C-E). We isolated $\mathrm{Ctsk}^{+}$cells from the Achilles tendon by FACS and found that the expression of Sufu was substantially decreased in the sorted $\mathrm{Ctsk}^{+}$cells from the Ctsk-CKO Rosa26-Ai9 mice compared with those from the CtskCtrl Rosa26-Ai9 mice (Figure 2F). And expression levels of the Hh target genes Gli1 and Ptch1 were much higher in the Ctsk-CKO Rosa26-Ai9 mice than in the Ctsk-Ctrl Rosa26-Ai9 mice (Figure 2F). The Ctsk ${ }^{+}$cells in the Ctsk-CKO Rosa26-Ai9 mice displayed increased chondrogenic markers (Sox9, Col2a1, Aggrecan) and osteogenic markers (Alp, Ocn, Opn) (Figure 2, G and H). Moreover, the $\mathrm{Ctsk}^{+}$cells showed decreased expression of the tendon-related genes $S c x, M k x$, and Tnmd (Figure 2I). Importantly, HO initiates in the midsubstance of the tendon, as shown by immunostaining for COLII and OPN of 4-, 5-, and 6-week-old Ctsk-CKO Rosa26Ai9 and control mice (Supplemental Figure 2A). The fact that the $\mathrm{COLII}^{+}$cells are organized in a row typical of tenocytes suggests the intrinsic changes to tenocytes (Figure 2C). To further confirm this finding, we crossed the Ctsk-CKO Rosa26-Ai9 mice with ScxG$F P$ mice. Cells expressing both the tenogenic marker ScxGFP and the chondrogenic markers COLII and aggrecan were observed in the $\mathrm{HO}$ at the midsubstance of the Achilles tendon (Figure 2, J and $\mathrm{K}$ ). As CD31 ${ }^{\text {hi }} \mathrm{Emcn}^{\text {hi }}$ (type $\mathrm{H}$ ) vessel formation is specifically coupled with new bone formation, type $\mathrm{H}$ vessels were found to be increased in the midsubstance of $\mathrm{HO}$ in the 20-week-old CtskCKO mice (Supplemental Figure 2, B and C) $(26,27)$.

In addition, the $\mathrm{Ctsk}^{+}$cells expressed the chondrogenic differentiation marker COLII, the osteogenic differentiation marker OPN/OCN (osteocalcin), and the osteoclast marker TRAP (tartrate-resistant acid phosphatase) in the patellar tendons of the 6-week-old Ctsk-CKO Rosa26-Ai9 mice (Supplemental Figure 2, D and E). Consistent with previous studies, Ctsk-Cre was expressed in a subset of perichondrial cells within the groove of Ranvier (Supplemental Figure 3A) (25). The Ctsk-Cre-expressing chon- droid cells in osteochondroma at the proximal tibia displayed various developmental stages, including $\mathrm{COLII}^{+}$proliferative chondrocytes and COLX ${ }^{+}$hypertrophic chondrocytes (Supplemental Figure 3, B and C). Taken together, these data indicate that the Ctsk-Cre-expressing cells at the tendons and ligaments contributed to the development of HO in this model.

Ctsk-Cre labels a unique subpopulation of TDPCs. The above research indicated that Ctsk-Cre may label a group of stem/progenitor cells in response to $\mathrm{Hh}$ signaling in the tendons and ligaments. These Ctsk-Cre-expressing cells in the tendinous insertions of the patella, quadriceps tendon, and Achilles tendon expressed the tenogenic marker $S c x$ after crossing with ScxGFP mice (Figure 3A and Supplemental Figure 4A). Then, 4 distinct populations, Ctsk $\operatorname{Scx}^{-}(84.9 \% \pm 14.3 \%), \mathrm{Ctsk}^{-} \mathrm{Scx}^{+}(2.1 \% \pm 1 \%), \mathrm{Ctsk}^{+} \mathrm{Scx}^{-}(10.2 \%$ $\pm 1.6 \%)$, and $\mathrm{Ctsk}^{+} \mathrm{Scx}{ }^{+}(2.9 \% \pm 1.9 \%)$, were identified by FACS analyses (Figure 3B). Moreover, the $\mathrm{Ctsk}^{+} \mathrm{Scx}^{+}$cells were showed to be enriched in the TDPC markers CD44, CD105, Nestin, and Sca1, as well as other stem/progenitor cell surface markers, such as CD24 and CD200 (Figure 3C and Supplemental Figure 4C). In vitro colony formation has been identified as a progenitor cell characteristic. For analysis of the colony-forming ability of the 4 populations, Ctsk-Scx, Ctsk ${ }^{+} \mathrm{Scx}^{-}, \mathrm{Ctsk}^{-} \mathrm{Scx}^{+}$, and $\mathrm{Ctsk}^{+} \mathrm{Scx}^{+}$cells were isolated and cultured. By culture day 7, the $\mathrm{Ctsk}^{+} \mathrm{Scx}^{+}$cells exhibited much higher colony-formation efficiency than the other cells (Figure 3, D and E). As previous studies have suggested that TGF- $\beta$ ligands can induce the expression of $S c x$, we also found that TGF- $\beta 3$ maintained $S c x$ expression in the $\mathrm{Ctsk}^{+} S \mathrm{cx}^{+}$cells in vitro (Figure 3F) (28-30). Next, we compared the multipotent differentiation potential of the 4 subpopulations for osteogenesis, adipogenesis, and chondrogenesis. Neither $\mathrm{Ctsk}^{-} \mathrm{Scx}^{-}$nor $\mathrm{Ctsk}^{-} \mathrm{Scx}^{+}$ tendon-derived cells showed multipotent differentiation potential in vitro. Interestingly, $\mathrm{Ctsk}^{+} \mathrm{Scx} \mathrm{x}^{+}$tendon-derived cells showed a higher capability of differentiating into all of the 3 lineages than $\mathrm{Ctsk}^{+} \mathrm{Scx}$ tendon-derived cells (Figure $3 \mathrm{G}$ and Supplemental Figure 4D). Taken together, these data indicated that Ctsk-Cre could label a unique subpopulation of TDPCs marked by ScxGFP, which represents a novel cell origin of HO.

Sufu-deficient tendon-derived cells exhibit enhanced chondrogenic and osteogenic differentiation. Previous histological analysis showed increased chondrocytes and osteoblasts in the tendons of the Ctsk-CKO mice, which prompted us to test whether Sufu was critical for the differentiation of TDPCs. We isolated and cultured $\mathrm{Ctsk}^{+}$cells from the Achilles tendons of the 4-week-old Ctsk-Ctrl Rosa-Ai9 mice and Ctsk-CKO Rosa-Ai9 mice. The Ctsk ${ }^{+}$cells from the Achilles tendons of the Ctsk-CKO Rosa-Ai9 mice showed enhanced chondrogenic differentiation (Figure 4A). Quantitative reverse transcriptase PCR (qRT-PCR) showed higher expression of chondrogenic genes (Sox9, Col2a1, Aggrecan) in the Sufu-deficient tendon-derived cells than in the control cells (Figure 4B). Tendon-derived cells from the 4-week-old Ctsk-CKO Rosa-Ai9 mice revealed enhanced ALP staining and higher supernatant ALP activity after 7 days of culture compared with the cells from the Ctsk-Ctrl Rosa-Ai9 mice (Figure 4, C and D). Sufu deficiency resulted in increased mineralization by alizarin red staining after 14 days of culture (Figure 4C). qRT-PCR showed higher expression of Hh target genes (Gli1, Gli2, Patch1) and osteogenic genes $(A l p, B s p, O c n, R u n x 2, O s x)$ in the Sufu-deficient tendon-derived 
A

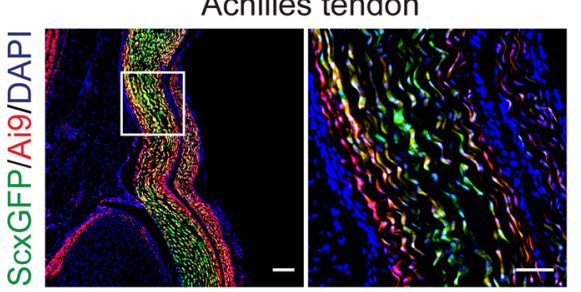

B

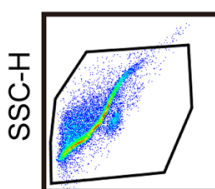

FSC-H

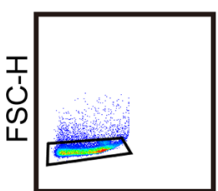

FSC-W

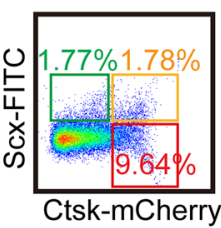

CD200

C
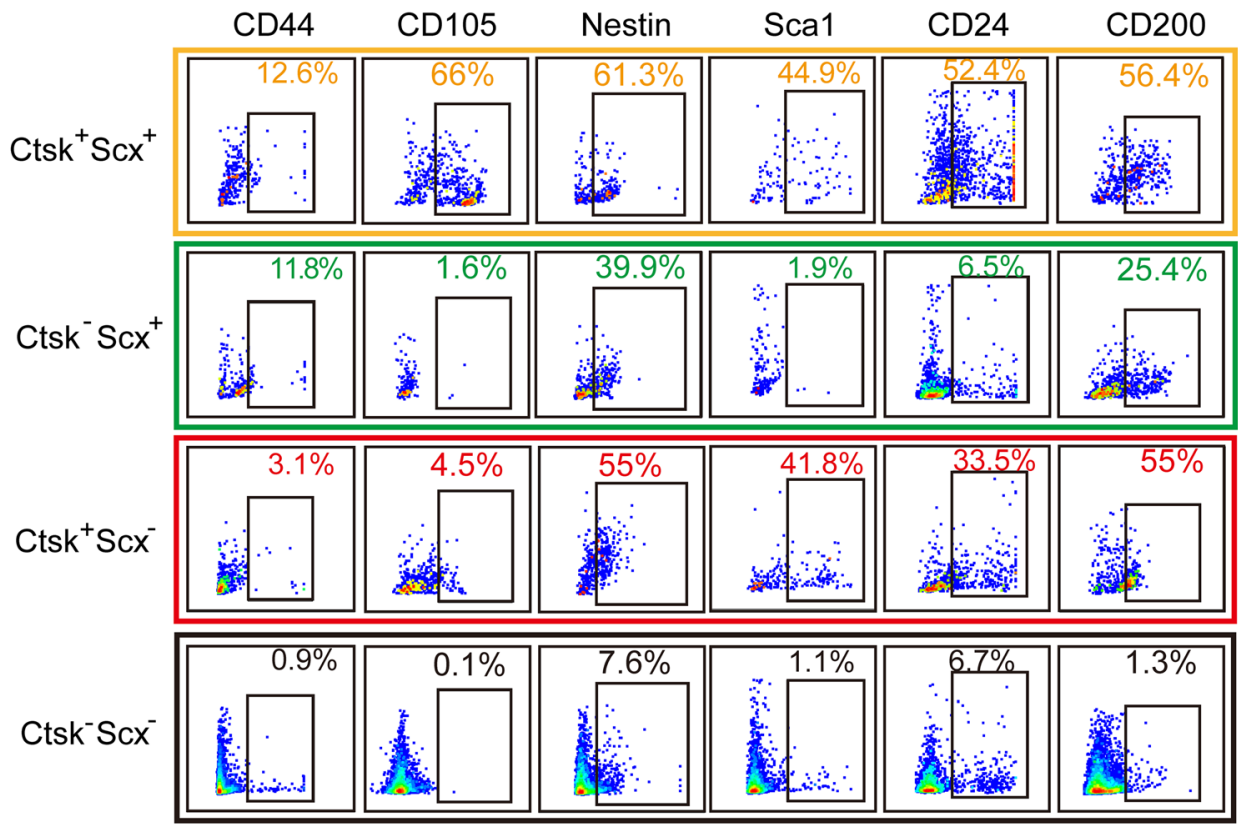

D

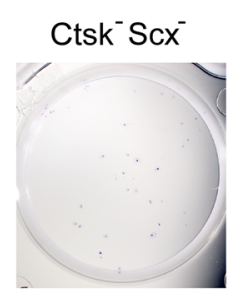

$\mathrm{Ctsk}^{+} \mathrm{Scx}^{-}$

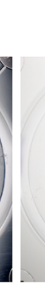

Ctsk $^{-} \mathrm{Scx}^{+}$

F

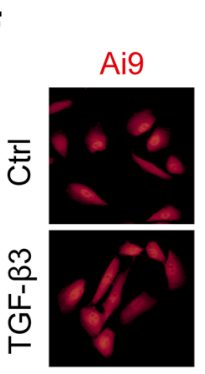

$\mathrm{Ctsk}^{+} \mathrm{Scx}^{+}$

ScxGFP

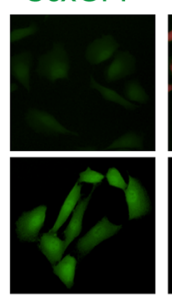

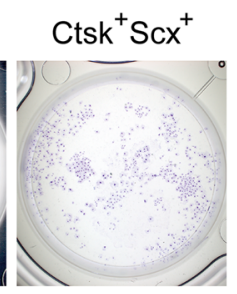

E

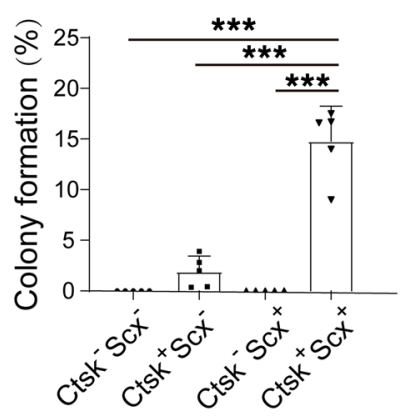

G

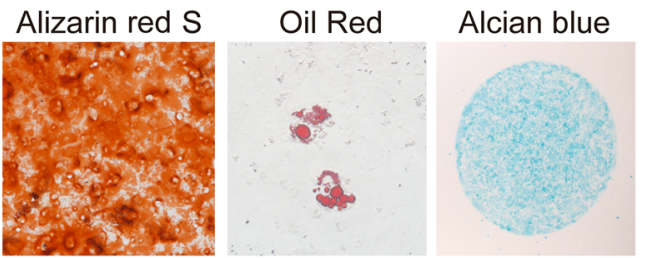

Safranin 0

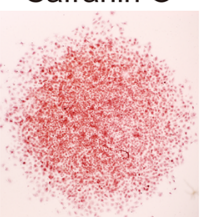

Figure 3. Ctsk-Cre labels a unique subpopulation of TDPCs. (A) Fluorescence imaging of the Achilles tendon of 2-week-old Ctsk-Cre Rosa26-Ai9 ScxCFP mice.

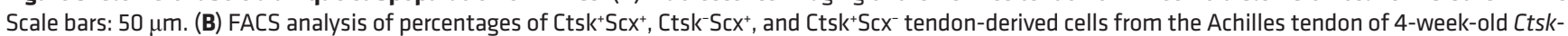
Cre Rosa26-Aig ScxGFP mice. Images are representative of 3 independent experiments. (C) FACS analysis of stem cell surface markers of Ctsk ${ }^{+}$Scx ${ }^{+}$, Ctsk-Scx ${ }^{+}$, and Ctsk ${ }^{+}$Scx tendon-derived cells from the Achilles tendon of 4-week-old Ctsk-Cre Rosa26-Aig ScxGFP mice. Images are representative of 3 independent experiments. (D) Colony formation assay using $\mathrm{Ctsk}^{-} \mathrm{Scx}, \mathrm{Ctsk}^{+} \mathrm{S} \mathrm{cx}^{-}, \mathrm{Ctsk}^{-} \mathrm{S} \mathrm{cx}^{+}$, and $\mathrm{Ctsk}^{+} \mathrm{S} \mathrm{cx}^{+}$tendon-derived cells from the Achilles tendon of 4-week-old Ctsk-Cre Rosa26-Ai9 ScxGFP mice. Colonies were stained with crystal violet after 7 days of culture $(n=5)$. (E) Colony numbers were counted, and colony-formation efficiencies in each group are shown $(n=5)$. Data are presented as the mean \pm SEM; 1-way ANOVA with Tukey's test was used for comparison of multiple groups; ${ }^{* * *} P<0.001$. (F) Fluorescence imaging of the cultured $C t s k^{+} S c x^{+}$TDPCs after 96 hours with or without TCF- $\beta 3$. (C) In vitro differentiation of sorted Ctsk ${ }^{+}$Scx $x^{+}$TDPCs into osteogenic lineage ( 3 weeks) by alizarin red S staining, adipogenic lineage (1 week) by Oil Red 0 staining, and chondrogenic lineage ( 2 weeks) by Alcian blue and Safranin 0 staining. 
A

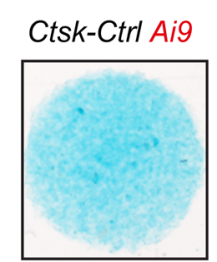

C Ctsk-Ctrl Ai9

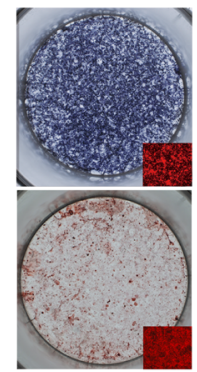

B
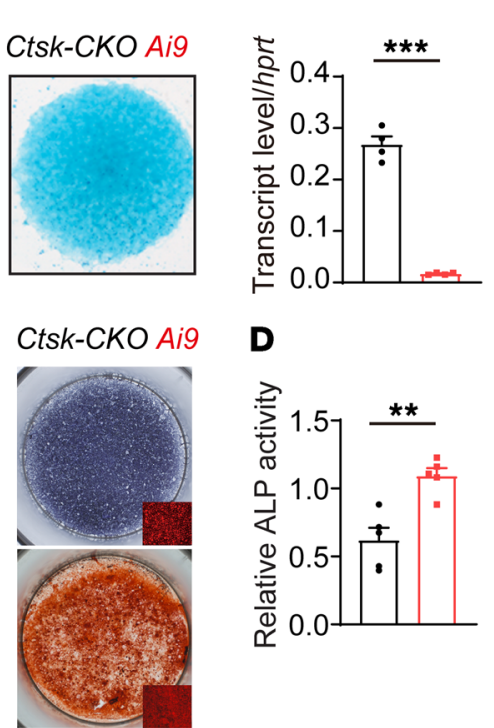

Sox9

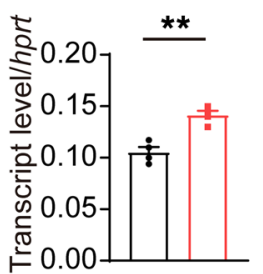

Col2a1

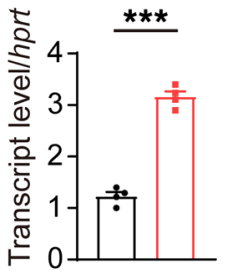

Aggrecan

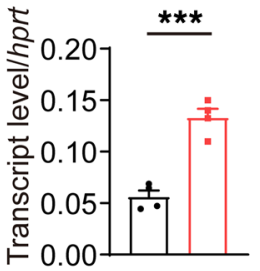

$\square$ Ctsk-Ctrl Ai9

$\square$ Ctsk-CKO Ai9
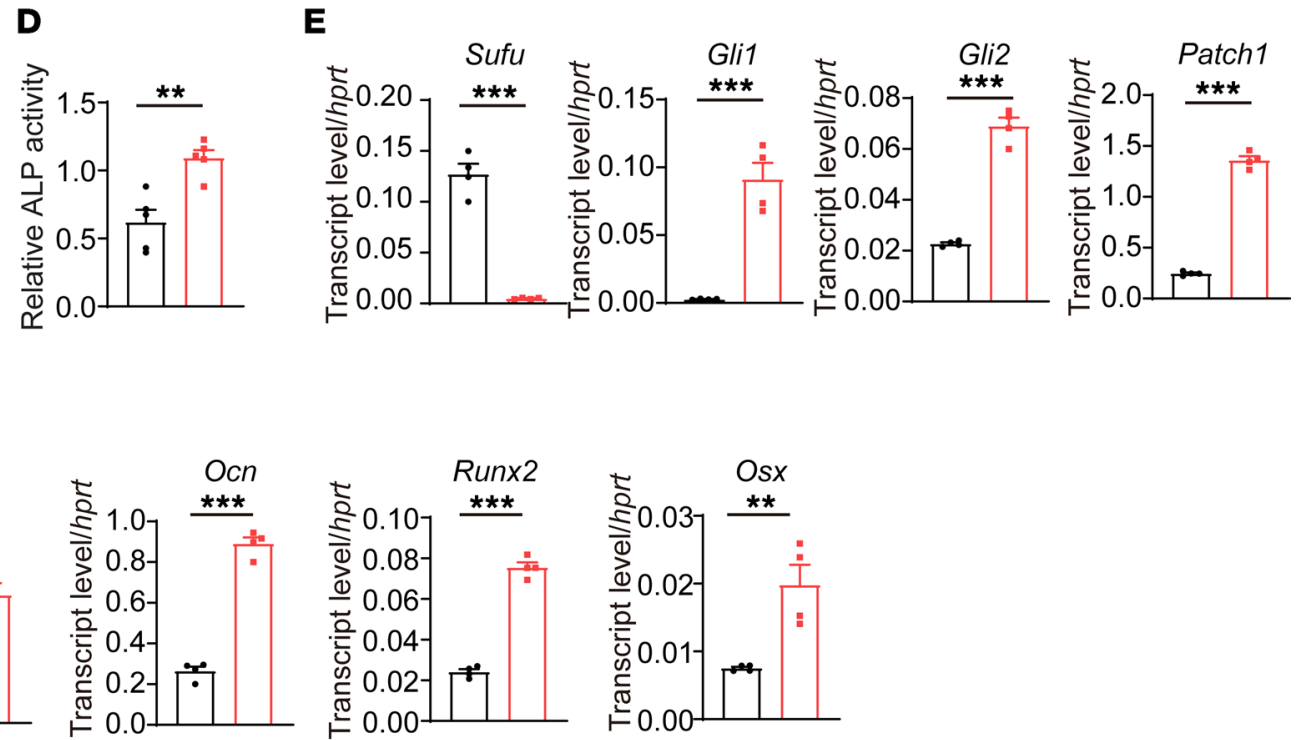

F
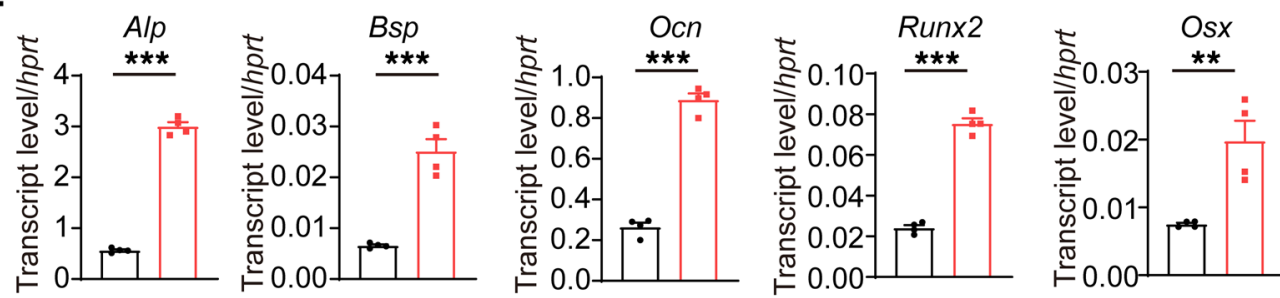

G

Ctsk-Ctrl Ai9

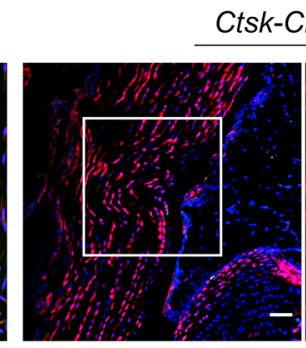

Ctsk-CKO Ai9

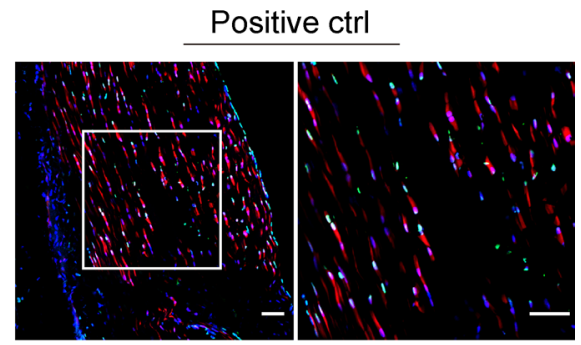

Figure 4. Sufu-deficient tendon-derived cells exhibit enhanced chondrogenic and osteogenic differentiation. (A) Chondrogenic differentiation of high-density sorted Achilles tendon cells from 4-week-old Ctsk-CKO Rosa26-Ai9 mice and Ctsk-Ctrl Rosa26-Aig mice cultured in chondrogenic medium was assessed by Alcian blue staining at day $10(n=4)$. (B) qRT-PCR analysis of gene markers of chondrogenesis. (C) Osteogenic differentiation analysis of Sufu-deficient tendon-derived cells and control cells by ALP staining (1 week) and alizarin red S staining ( 2 weeks) $(n=5)$. Insets represent the fluorescence signals of cells after differentiation. (D) Quantification of ALP activity relative to cell numbers. $n=5$ per group. (E and $\mathbf{F}$ ) Osteogenic differentiation of sorted Achilles tendon cells from 4-week-old Ctsk-CKO Rosa26-Ai9 mice and Ctsk-Ctrl Rosa26-Ai9 mice. Gene expression analysis of markers of Hh signaling and osteogenesis by qRT-PCR. (C) TUNEL assays revealed apoptosis of the Achilles tendon from 4-week-old Ctsk-CKO Rosa26-Ai9 mice and control mice. $n=3$ per group. DNase I was used as positive control. Scale bars: $50 \mu \mathrm{m}$. Results are presented as the mean $\pm \mathrm{SEM}$; unpaired $t$ test, ${ }^{* *} P<0.01,{ }^{* * *} P<0.001$.

cells than in the control cells (Figure 4, E and F). TUNEL was performed to determine whether Sufu deficiency could affect the apoptosis of TDPCs. However, the Achilles tendons of both the 4-week-old Ctsk-Ctrl Rosa-Ai9 mice and the Ctsk-CKO Rosa-Ai9 mice showed rare TUNEL-positive cells (Figure 4G). These data suggest that Sufu deficiency leads to enhanced chondrogenic and osteogenic differentiation, but not apoptosis of TDPCs.

Gli1/Gli2 deficiency prevents HO progression in Ctsk-CKO mice. In canonical Hh signaling, SUFU deficiency led to dysregulation of Smoothened (SMO) to regulate mobilization of the zinc finger transcription factor GLI2. GLI2 translocates to the nucleus from cilia and transactivates the GLI1 promoter. GLI1 and GLI2 directly activate transcription of Hh target genes (31). To determine whether activated $\mathrm{Hh}$ signaling is essential in $\mathrm{HO}$ and osteochondroma development, we deleted Gli1 or Gli2 in the Ctsk-CKO mice by crossing Gli1 ${ }^{\text {lacZ/lacZ }}$ knockin mice or Gli2 $2^{f / f l}$ mice, and the knockout efficiency was verified by Western blot (Supplemental Figure 5, A and B). X-ray and $\mu \mathrm{CT}$ radiographs demonstrated that $\mathrm{HO}$ in the periarticular and Achilles tendons was almost rescued in the 20-week-old Ctsk-CKO Glillacz/lacz and Ctsk-CKO Gli2 $2^{f / f l}$ double-knockout mice (Figure 5A and Supplemental Figure 5C). Osteophytes in the periarticular region of the double-knockout 
A
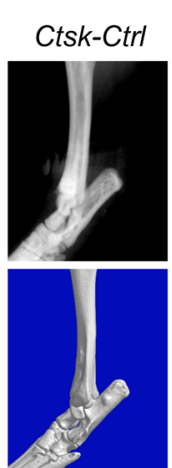
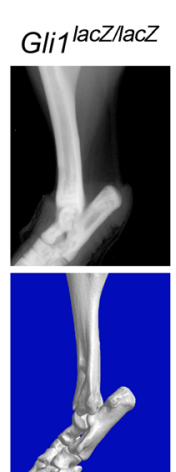

Ctsk-Cre
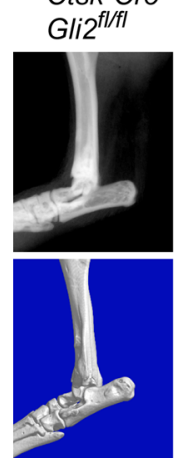

Ctsk-CKO
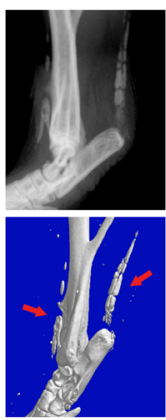

Ctsk-CKO Ctsk-CKO

Glit 1acZlacz
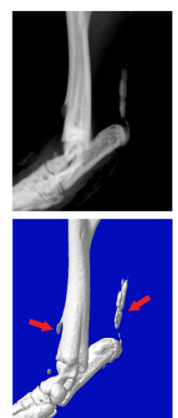

$\mathrm{Gli}^{\mathrm{fl|f|} \mid \mathrm{K}}$
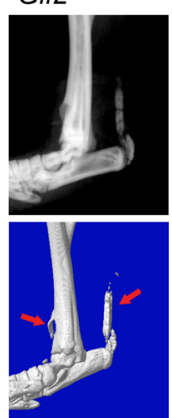

B

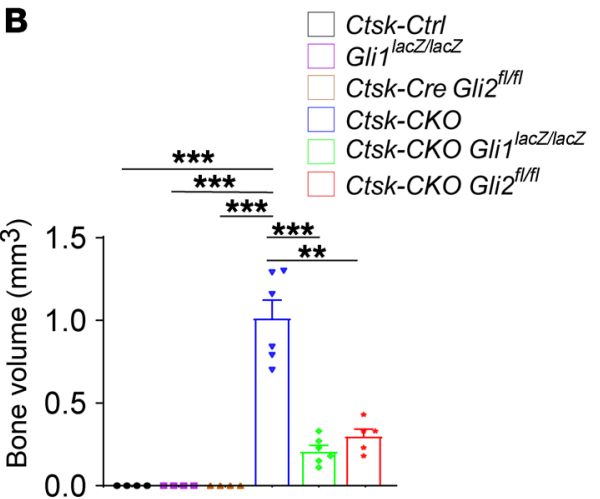

C

C Ctsk-Ctrl

Gli1/acZlacz

Ctsk-Cre Gli2 ${ }^{f / f f l}$

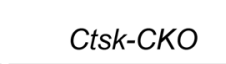

Ctsk-CKO
Gli1lacZlacz

Ctsk-CKO
Gli fliffl
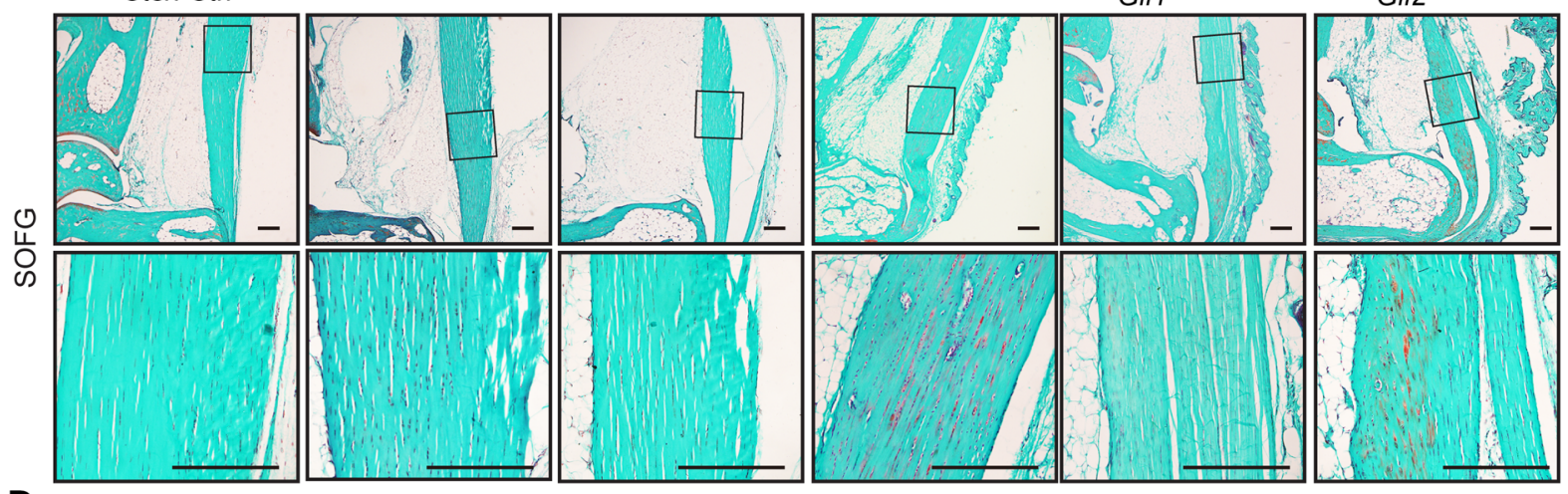

D
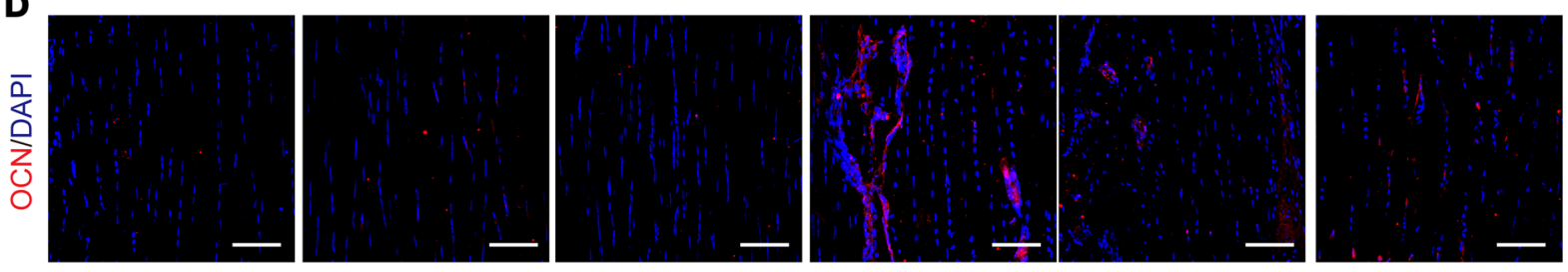

Figure 5. Gli1/Gli2 deficiency prevents HO progression in Ctsk-CKO mice. (A) X-ray and $\mu \mathrm{CT}$ radiographs show HO formation of the Achilles tendon of CtskCtrl, Gliitlacz/lacz, Ctsk-Cre Cliz fl/fl, Ctsk-CKO, Ctsk-CKO Gli lacz/lacz, and Ctsk-CKO Gli2fl/fl mice. (B) Quantitative analysis of the HO volume around the ankles of

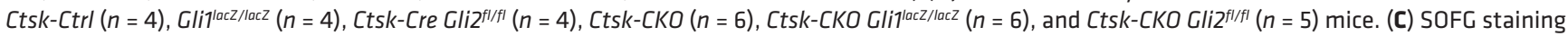

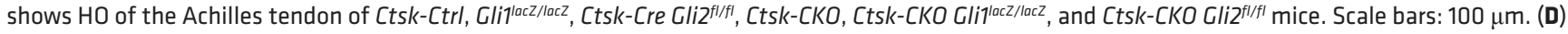

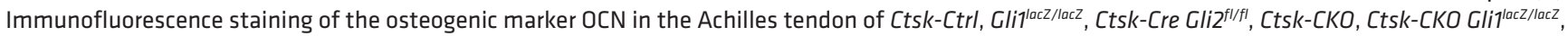
and Ctsk-CKO Gli2f/fl mice. Scale bars: $50 \mu \mathrm{m}$. Data are presented as the mean \pm SEM; 1-way ANOVA with Tukey's test was used for comparison of multiple groups; ${ }^{* *} P<0.01$, ${ }^{* *} P<0.001$.

mice disappeared (Supplemental Figure 5C), and the HO volumes of the ligaments around ankle joints and Achilles tendons were significantly decreased (Figure 5, A and B). SOFG staining showed that no $\mathrm{HO}$ occurred in the periarticular region of the double-knockout mice, and osteochondromas were also rescued (Supplemental Figure 5D). The hind paws of these 2 double-knockout mice appeared normal (Supplemental Figure 5E).The Safranin Opositive chondrocytes and OCN-positive osteoblasts in the Achilles tendons of the double-knockout mice were decreased (Figure $5, \mathrm{C}$ and D). These results indicate that a stabilized level of Hh signaling activity is essential for the maintenance of progenitors in ligaments, tendons, and perichondrium.

Pharmacological inhibition of Hh signaling by JQ1 ameliorates HO progression in the Ctsk-CKO mice. Gant61 can block the transcription of Gli1, which has been used in the treatment of various types of Hh-overactive tumors in mice $(32,33)$. To verify the effects of Gant61 on the Sufu-deficient TDPCs, we treated the sorted 4-week-old tendon-derived cells from the Ctsk-CKO Rosa26-Ai9 mice with gradient concentrations of Gant61. As expected, the expression of Hh target genes (Gli1, Gli2, Patch1) decreased after treatment (Supplemental Figure 6A). Gant61 treatment inhibited the chondrogenic and osteogenic differentiation of the tendon-derived cells from Ctsk-CKO Rosa-Ai9 mice in vitro (Figure 6, A-D; and Supplemental Figure 6B). The Ctsk-CKO mice were intraperitoneally injected with $50 \mathrm{mg} / \mathrm{kg}$ Gant61 every 2 days for 3 weeks starting at the age of 4 weeks in vivo. However, the Ctsk-CKO mice treated with Gant61 showed no obvious change compared with the Ctsk-Ctrl mice (Supplemental Figure 6C). JQ1, a small-molecule inhibitor targeting BRD4, has been reported to inhibit the transcription of GLI1 and GLI2 and treat Hh-driven tumors with 
resistance to SMO antagonists (34). We found that JQ1 could dramatically inhibit the transcription of $\mathrm{Hh}$ target genes of the tendon-derived cells from the Ctsk-CKO Rosa-Ai9 mice (Supplemental Figure 6A). This molecule also inhibited the chondrogenic and osteogenic differentiation of the tendon-derived cells of the Ctsk-CKO Rosa-Ai9 mice (Figure 6, A-D; and Supplemental Figure 6B). Then the Ctsk-CKO mice were injected intraperitoneally with $50 \mathrm{mg} / \mathrm{kg}$ JQ1 daily for 3 weeks starting at the age of 4 weeks. The mobility of the treated Ctsk-CKO mice was improved significantly. Both osteochondroma and $\mathrm{HO}$ in the periarticular and Achilles tendons were notably ameliorated by JQ1, as determined by $\mu \mathrm{CT}$ radiographs (Figure $6, \mathrm{E}$ and $\mathrm{F}$ ). In addition, this dosage of JQ1 showed no apparent detrimental effects on the growth of long bones of the treated control mice (Figure 6E). The HO areas of the patellar tendon and Achilles tendon were decreased notably as assessed by SOFG staining (Figure 6G and Supplemental Figure $6 \mathrm{D}$ ). The chondrocytes within osteochondroma were also decreased after JQ1 treatment (Supplemental Figure 6D).

In conclusion, these results suggest that enhancement of $\mathrm{Hh}$ signaling by deletion of Sufu in Ctsk-expressing cells can induce $\mathrm{HO}$ in tendons and ligaments and that JQ1 might function as a potential therapeutic drug in the treatment of Hh-driven $\mathrm{HO}$.

\section{Discussion}

Tendon progenitor cells had so far been studied in vitro $(13,14$, 29). The identification of tendon progenitor cells in vivo and study of these cells are significant. We report, for the first time to our knowledge, that Ctsk-Cre could label a subpopulation of TDPCs that contribute to $\mathrm{HO}$ in vivo. Previous studies demonstrated that Ctsk is also a marker of mature osteoclasts, chondroprogenitors in the perichondrial groove of Ranvier, and periosteal mesenchymal stem/progenitor cells (22-25). On the basis of our data, $\mathrm{Ctsk}^{+}$tendon-derived cells are also rich in TDPCs in combination with an ScxGFP reporter. Previous studies have shown that tendon progenitor cells marked by $S c x$ contribute to $\mathrm{HO}$ in tendons and ligaments $(5,6)$. We identified a unique population, $\mathrm{Ctsk}^{+} \mathrm{Scx}^{+} \mathrm{TDPCs}$, from $\mathrm{Scx}^{+}$progenitor cells by $C t s k$. The $\mathrm{Ctsk}^{+} \mathrm{Scx}^{+} \mathrm{TDPCs}$ showed stronger clonogenic abilities and expressed higher levels of tendon stem/progenitor cell markers than other populations. The $\mathrm{Ctsk}^{+} \mathrm{Scx}^{+}$TDPCs also showed higher mutipotential abilities and tended to differentiate into chondrocytes and osteoblasts with the overactive Hh signaling.

Hh signaling has been reported to be involved in HO (1). A recent study showed that Gli1-labeled adult mesenchymal stem/ progenitor cells contributed to endochondral $\mathrm{HO}$ (7). Ablation of Hh signaling can inhibit the $\mathrm{HO}$ process in an $M k x$-deficient mouse model and a trauma-induced $\mathrm{HO}$ mouse model $(35,36)$. Activation of Hh signaling is involved in intramembranous ossification in a POH mouse model (Prx1-Cre Gnas ${ }^{f / f l}$ mice) (19). Our mouse model provides direct evidence that dysregulation of $\mathrm{Hh}$ signaling leads to chondrogenic differentiation of TDPCs. The fact that activation of Hh signaling induces 2 different HO phenotypes may be due to the different cell types that Hh signaling affects. Our data show that activation of $\mathrm{Hh}$ signaling with abnormal Gli1 expression induces Sox9 expression in the midsubstance. This finding indicates that abnormal Hh signaling has a major impact on the cell properties and functions of TDPCs in tendons and ligaments.
A limitation of this study is that the Ctsk-Cre mouse is a noninducible mouse line. We cannot distinguish when these progenitor cells are marked. A CreERT2 mouse line is needed to address this question. With the CreERT2 mouse line, we can also define the role of Ctsk-lineage cells involved in trauma-induced HO and the distribution pattern of Ctsk-lineage cells in HO stages by tracing.

Our study suggests that the cell fate of tendon progenitor cells is regulated by $\mathrm{Hh}$ signaling. The fact that $\mathrm{Scx}^{+}$tendon progenitor cells expressed the chondrogenic markers in HO provides additional evidence that tendon progenitor cells can differentiate into chondrocytes. As in previous studies, disturbance of Bmp signaling can also induce chondrogenic differentiation of tendon progenitor cells $(5,6)$. However, we cannot exclude the possibility that some $\mathrm{HO}$ cells are externally recruited $\mathrm{Ctsk}^{+}$cells, especially during new bone formation for vessel invasion.

Ptpn11 deletion in Ctsk-Cre-expressing perichondrium cells causes metachondromatosis, a rare inherited disorder featuring multiple exostoses and enchondromas, which results from enhanced Hh signaling (25). Our model provides evidence that upregulation of Hh signaling can lead to the formation of osteochondromas that originate from the groove of Ranvier. However, enchondroma was not observed in our model instead of heterotopic ossified lesions. A previous study reported that IHH/ PTHrP signaling contributes to the genesis of enchondromas by delaying terminal chondrocyte differentiation (37). The deletion of Ptpn11 leads to an increase in the Hh ligand Ihh (25). Sufu deletion can lead to activation of Hh signaling independent of Hh ligand $(31,38)$. The difference between the Ptpn11 and Sufu mouse models indicates the difference between ligand-dependent Hh signaling and cell-autonomous Hh signaling induced by deletion of Sufu. Interestingly, we found that upregulation of $\mathrm{Hh}$ signaling by inactivation of Sufu in the Ctsk-Cre-expressing cells could induce osteochondroma and progressive $\mathrm{HO}$ in tendons and ligaments, while overactive Bmp signaling can also induce these phenotypes (6). Inhibition of Hh signaling by targeting of Gli1 can prevent the HO process of BMP4 and trauma-induced HO (35). It is likely that all HO cases share a common pathway that leads to the conversion of mesenchymal progenitor cells to osteoblast lineages or chondrocytes from other cell lineages. The mechanism of crosstalk between them remains to be further determined.

Hh inhibitors including SMO antagonists and GLI antagonists were reported to treat different types of Hh-driven cancers $(39,40)$. Our study showed that JQ1, a BET inhibitor that has been proven effective in treating Hh-driven tumors resistant to SMO antagonists (34), can ameliorate HO in the Ctsk-Cre Sufu $u^{f / f l}$ mouse model. A previous study showed that upregulation of $\mathrm{Hh}$ signaling is found in human HO samples of the posterior longitudinal ligament and that $\mathrm{Hh}$ signaling promotes chondrocyte differentiation in endochondral ossification (41). Moreover, Hh signaling has been reported to contribute to endochondral $\mathrm{HO}$ of tendons in an injury-induced transgenic mouse model, and $\mathrm{HO}$ was prevented by ablation of Hh signaling $(7,35)$. JQ1 could inhibit the enhanced chondrocyte differentiation of TDPCs caused by upregulation of Hh signaling in vitro and in vivo. Our study indicates that JQ1 may be a new therapeutic drug for endochondral $\mathrm{HO}$ in ligaments and tendons. 
A Ctsk-Ctrl Ai9
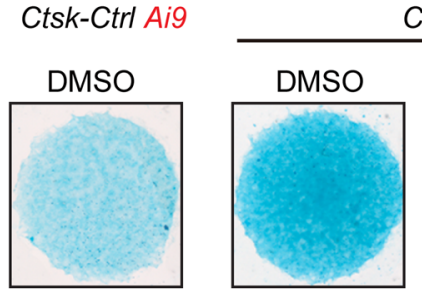

Ctsk-CKO Aig
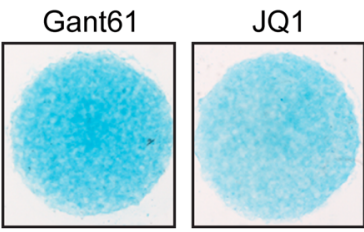

C Ctsk-Ctrl Ai9
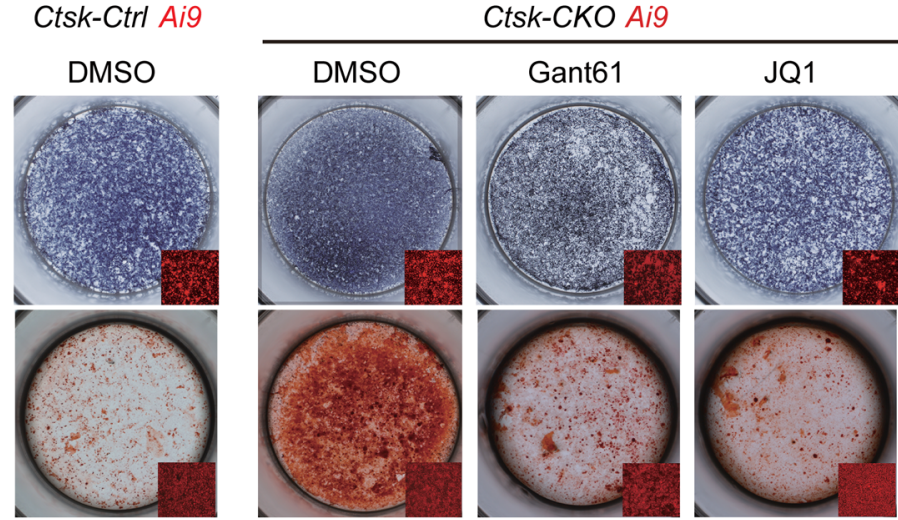

B Col2a1

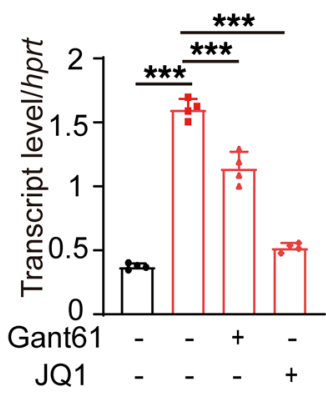

Aggrecan

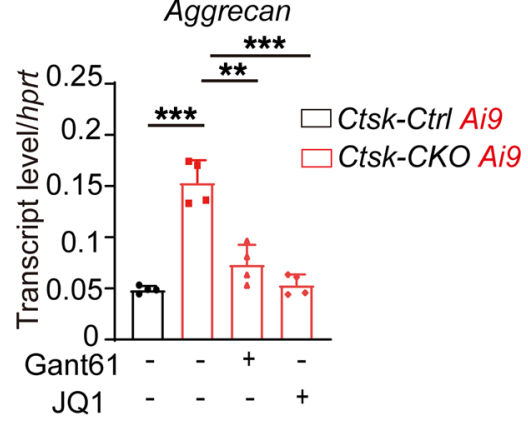

E

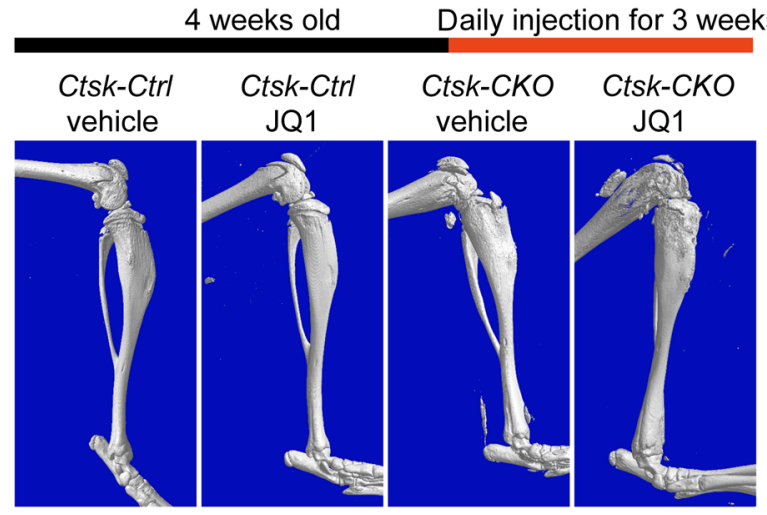

D
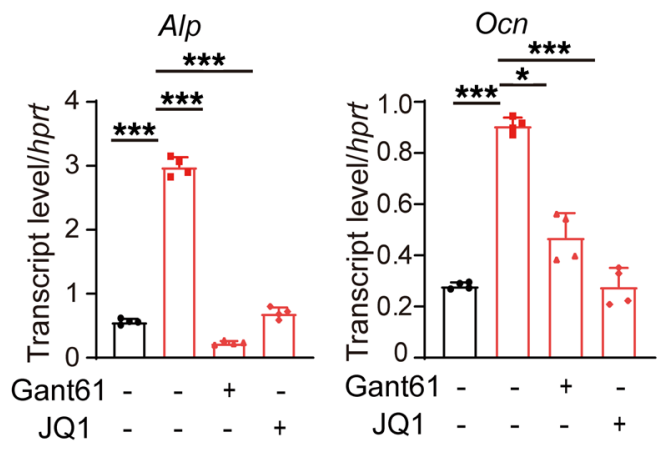
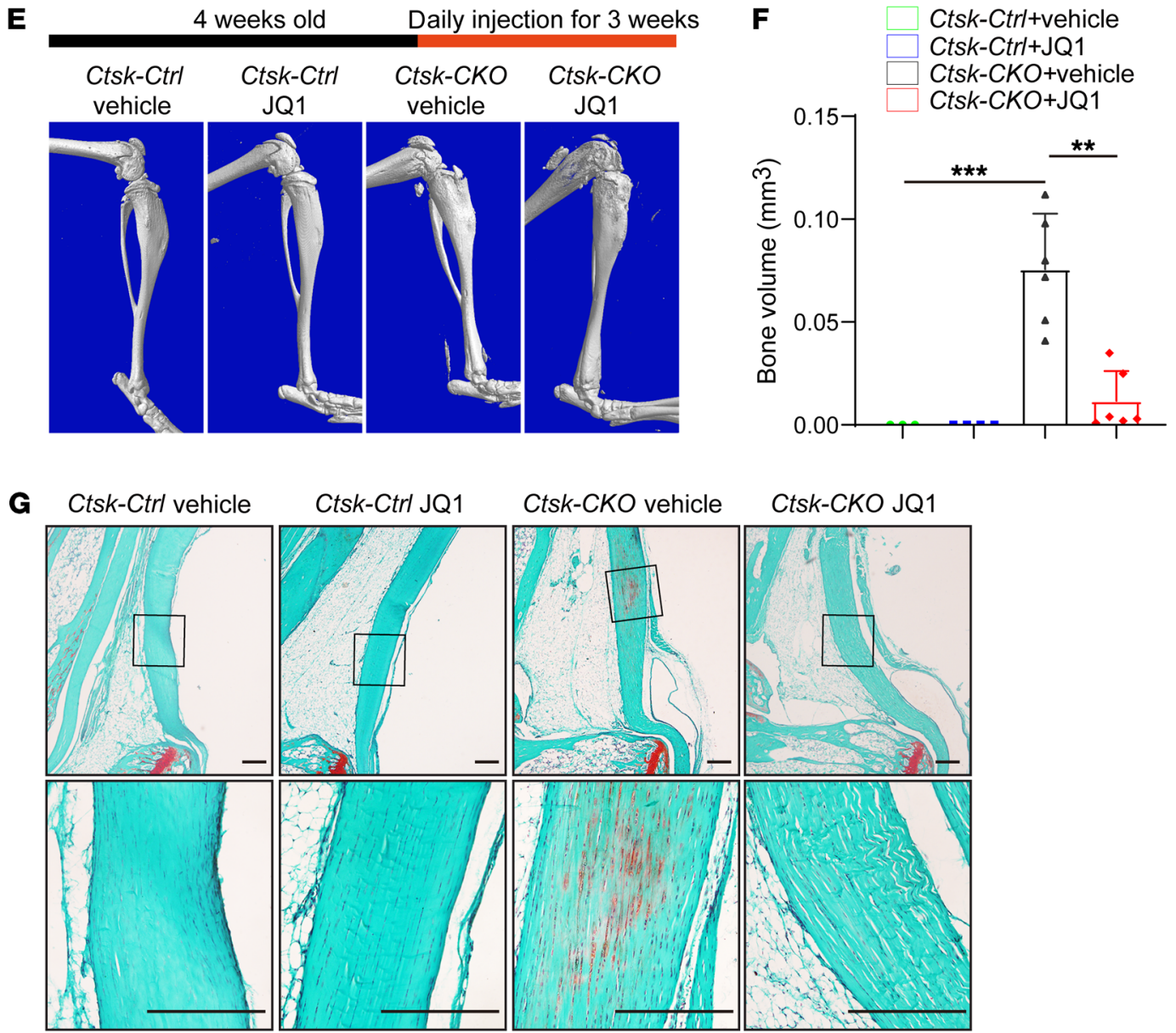
Figure 6. Pharmacological inhibition of Hh signaling by JQ1 ameliorates HO progression in Ctsk-CKO mice. (A) Alcian blue staining of high-density sorted Achilles tendon cells from 4-week-old Ctsk-CKO Rosa26-Aig mice and Ctsk-Ctrl Rosa26-Ai9 mice cultured in chondrogenic medium treated with Gant61 and JQ1 at day $10(n=4)$. (B) qRT-PCR analysis of gene markers of chondrogenesis. (C) ALP staining (day 7) and alizarin red S staining (day 14) of sorted Ctsk ${ }^{+}$cells from the Achilles tendon of CtskCKO Rosa26-Ai9 mice and Ctsk-Ctrl Rosa26-Ai9 mice treated with Gant61 and JQ1 in osteogenic medium for 14 days $(n=4)$. Insets represent the fluorescence signals of the cells after differentiation. (D) Gene expression of markers of $\mathrm{Hh}$ signaling and osteogenic differentiation after Gant61 and JQ1 treatment. (E) $\mu \mathrm{CT}$ radiographs showed the $\mathrm{HO}$ of $C t s k-C t r l$ mice with vehicle treatment $(n=3)$, Ctsk-Ctrl mice with JQ1 treatment $(n=4)$, Ctsk-CKO mice with vehicle treatment $(n=6)$, and Ctsk-CKO mice with JQ1 treatment $(n=6)$. (F) Quantitative analysis of the whole HO volume of Ctsk-Ctrl mice treated with vehicle $(n=3)$, Ctsk-Ctrl mice treated with JQ1 $(n=4)$, Ctsk-CKO mice treated with vehicle $(n=6)$, and Ctsk-CKO mice treated with JQ1 $(n=6)$. (G) SOFG staining of the Achilles tendon of CtskCtrl and Ctsk-CKO mice with JQ1 or vehicle treatment. Images are representative of 4 different sets of experiments. Scale bars: $100 \mu \mathrm{m}$. Data are presented as the mean \pm SEM; 1-way ANOVA with Tukey's test was used for comparison of multiple groups; ${ }^{*} P<0.05$, ${ }^{* *} P<0.01$, ${ }^{* * *} P<0.001$.

\section{Methods}

Mice. Sufu ${ }^{f l f l}$ mice containing loxP sites flanking exons 4-8 of Sufu and Gli1 ${ }^{\text {lacz/lacz }}$ knockin mice were generated in-house $(42,43)$. Gli $^{f / / f l}$ mice were provided by Zunyi Zhang (Hangzhou Normal University, Hangzhou, China) (44). ScxGFP transgenic mice were obtained from Hongwei Ouyang (Zhejiang University, Hangzhou, China) (45). Ctsk-Cre mice were provided by S. Kato (University of Tokyo, Tokyo, Japan) (24). Ai9 reporter mice were provided by Zilong Qiu (Chinese Academy of Sciences, Shanghai, China) (46). All mice analyzed were purified and maintained on the $129 / \mathrm{Sv}$ background. Sex-matched littermate controls were used in all analyses. Both male and female mice were analyzed. All mice were bred and maintained under specific pathogen-free conditions.

Radiographic assessment. All mice were euthanized by carbon dioxide, and the hind limbs or whole bodies were kept in $70 \%$ ethanol. The skeletons were analyzed using Faxitron MX-20 for x-ray image analysis.

For $\mu \mathrm{CT}$ analysis, the hind limbs or whole bodies isolated from the same age and sex were fixed in 70\% ethanol and scanned using SkyScan 1272 (Bruker, Kartuizersweg, Belgium) at a resolution of $18 \mu \mathrm{m}$. Then 3D reconstruction was performed by the same threshold.

Histology analysis. For paraffin sections, femurs and tibiae were fixed in precooled $4 \%$ paraformaldehyde for 48 hours at $4^{\circ} \mathrm{C}$, and decalcified in 10\% EDTA (pH 7.5). After PBS washing, the specimens were dehydrated with $70 \%$, 95\%, and $100 \%$ ethanol, embedded with paraffin after xylene immersion, and cut into $7-\mu \mathrm{m}$-thick sections. The sections were stained with $\mathrm{H} \& \mathrm{E}$ and Safranin O/fast green (SOFG) following standard steps (47).

For frozen sections, the hind limbs and tendons were fixed in precooled $4 \%$ paraformaldehyde for 48 hours at $4^{\circ} \mathrm{C}$. After complete decalcification in 10\% EDTA, they were dehydrated in 30\% sucrose overnight and then embedded with OCT compound (Tissue-Tek, 4583) and sectioned at $10 \mu \mathrm{m}$ thickness using a Leica CM3050 S freezing microtome.

Immunofluorescence. Frozen sections were air-dried and rehydrated with PBS, blocked, and permeabilized with $10 \%$ horse serum and $0.2 \%$ Triton $\mathrm{X}-100$ in PBS at room temperature for 1 hour. The sections were then probed with the following primary antibodies overnight at $4^{\circ} \mathrm{C}$ : goat anti-OPN (R\&D Systems, AF808; 1:400), goat anti-CD31 (R\&D Systems, AF3628; 1:200), mouse anti-COL2 (Abcam, ab185430; 1:200), rabbit anti-TRAP (Abcam, ab185716; 1:200), rabbit anti-osteocalcin (Abcam, ab93876; 1:200), rabbit anti-Col10a1 (ABclonal, a6889; 1:200), rabbit anti-aggrecan (MilliporeSigma, ab1031x; 1:100), and rat anti-EMCN (Santa Cruz Biotechnology, sc-65495; 1:200). Fluorescence-labeled secondary antibodies (1:1000) were incubated for 1 hour under shielding from light at room temperature after washing with PBS. Nuclei were stained with DAPI (MilliporeSigma, D9542). Sections were mounted using fluorescence mounting medium (Dako, S3023). Imaging was performed with an Olympus FV1200 confocal microscope.

Cell sorting and culture. Primary tendon-derived cells were isolated from the Achilles tendons of 4-week-old mice. After the tendon sheath was dissected, the tendon was cut into pieces and digested with collagenase II (1.5 mg/mL; MilliporeSigma) and dispase II $(2 \mathrm{mg} / \mathrm{mL}$; Roche) for 30 minutes at $37^{\circ} \mathrm{C}$. Digestion solution was collected and centrifuged at $500 \mathrm{~g}$ for 5 minutes. The isolated cells were cultured in a 24 -well plate with $\alpha$-MEM (Corning) containing $10 \%$ FBS. Then the fluorescence-labeled tendon-derived cells were sorted by Aria SORP.

FACS analysis. For the analysis of the cells from Achilles tendons of Ctsk-Cre Rosa26-Ai9 ScxGFP mice, a previously described method was used $(13,23)$. After RBCs among the acquired tendon-derived cells were removed by RBC lysis buffer (Beyotime, C3702), the acquired tendon-derived cells were stained with APCanti-mouse CD24 (BioLegend, 138505), APC-anti-mouse Nestin (BioLegend, 655108), APC-anti-CD44 (BioLegend, 559250), APC-anti-mouse CD200 (BioLegend, 123809), PE/Cy7-antimouse CD105 (BioLegend, 120409), and APC-anti-Sca1 (eBioscience, 17-5981-81). After PBS washes, flow cytometric analysis was conducted and analyzed using Beckman CytoFlex FCM.

Colony formation assay and in vitro multipotent differentiation. For the colony formation assay, approximately 20 cells of sorted Ctsk-Scx, $\mathrm{Ctsk}^{-} \mathrm{Scx}^{+}, \mathrm{Ctsk}^{+} \mathrm{S} \mathrm{cx}^{-}$, and $\mathrm{Ctsk}^{+} \mathrm{Scx}^{+}$cells from the Achilles tendons of the Ctsk-Cre Rosa26-Ai9 ScxGFP mice were cultured for 7 days. In vitro multipotent differentiation toward osteogenesis, adipogenesis, and chondrogenesis was performed as previously described $(13,23)$. For osteogenesis, briefly, approximately $2 \times 10^{4}$ cells were seeded per well in 96-well plates in $\alpha$-MEM (Corning) supplemented with $10 \%$ FBS and $1 \%$ penicillin/streptomycin (Thermo Fisher Scientific). After 12 hours, the medium was switched to osteogenic differentiation medium including $50 \mu \mathrm{g} / \mathrm{mL}$ ascorbic acid (MilliporeSigma) and $1 \mathrm{mg} / \mathrm{mL}$ glycerophosphate (MilliporeSigma). The medium was changed every 2 days. After 21 days of induction, the osteogenic differentiation ability was determined by alizarin red S staining.

For adipogenesis, the differentiation medium contained solution A and solution B. Solution A contained $\alpha$-MEM with 10\% FBS, 50 $\mathrm{mM}$ dexamethasone (MilliporeSigma), $100 \mathrm{nM}$ rosiglitazone (MilliporeSigma), $500 \mathrm{nM}$ 3-isobutyl-1-methylxanthine (IBMX) (MilliporeSigma), $10 \mathrm{mg} / \mathrm{mL}$ insulin (MilliporeSigma), and 1\% penicillin/ streptomycin (MilliporeSigma). Adipogenic differentiation was determined by $0.5 \%$ Oil Red O staining (MilliporeSigma) and BODIPY (Invitrogen) after 7 days of induction.

For chondrogenesis, briefly, cells were harvested and resuspended in $\alpha$-MEM supplemented with $10 \%$ FBS and $1 \%$ penicillin/streptomycin. Droplets $(15 \mu \mathrm{L})$ containing $2.5 \times 10^{5}$ cells were carefully placed 
in the middle of each well of a 24-well plate. After cells adhered at $37^{\circ} \mathrm{C}$ for $2-4$ hours, $500 \mathrm{~mL}$ of chondrogenic medium including $1 \%$ insulin-transferrin-selenium solution (ITS, MilliporeSigma), $10 \mathrm{ng} /$ $\mathrm{mL}$ TGF- $\beta 3$ (PeproTech), $100 \mathrm{nM}$ dexamethasone (MilliporeSigma), $40 \mu \mathrm{g} / \mathrm{mL}$ proline (MilliporeSigma), $50 \mu \mathrm{g} / \mathrm{mL}$ L-ascorbic acid 2-phosphate (MilliporeSigma), and $1 \mathrm{mM}$ sodium pyruvate (Thermo Fisher Scientific) was added. The medium was changed every 2 days. Micromass cultures were stained with Alcian blue at day 10.

Quantification of relative ALP activity. For quantitative analysis of the relative ALP activity, cells cultured after 7 days were incubated with alamarBlue (Thermo Fisher Scientific) for 4 hours at $37^{\circ} \mathrm{C}$, and the cellularity was determined using EnVision (PerkinElmer) at $580 \mathrm{~nm}$. Then the supernatants were removed, cells were incubated with ALP substrate solution containing $6.5 \mathrm{mM} \mathrm{Na}_{2} \mathrm{CO}_{3}, 18.5 \mathrm{mM} \mathrm{NaHCO}_{3}, 2 \mathrm{mM}$ $\mathrm{MgCl}_{2}$, and $1 \mathrm{mg} / \mathrm{mL}$ phosphatase substrate (MilliporeSigma) for 30 minutes, and ALP activities were determined with EnVision at $405 \mathrm{~nm}$.

TUNEL. TUNEL assays for apoptosis testing were performed as described by Promega.

Real-time RT-PCR analysis. Total RNA was prepared using TRIzol (MilliporeSigma) and was reverse-transcribed into cDNA with the PrimeScript RT Reagent Kit (TaKaRa). Real-time quantitative PCR was performed with the Bio-Rad CFX96 system. The sequences of oligonucleotides used for quantitative PCR are listed in Supplemental Table 1.

Western blot. Cells from Achilles tendons of mice were homogenized in cold lysis buffer supplemented with protease inhibitor cocktails (MedChemExpress, HY-K0010). Proteins were separated by SDS-PAGE and transferred to PVDF membrane. After blocking with $5 \%$ Non-Fat Powdered Milk (Sangon Biotech, A600669) in 1× TBST buffer, membranes were hybridized at $4^{\circ} \mathrm{C}$ overnight with the following primary antibodies: Gli1 (Cell Signaling Technology, 2534S; 1:1000) and Gli2 (Abcam, ab26056). Then, the membranes were incubated with HRP-conjugated secondary anti-rabbit IgG (Dako, P0217; 1:5000) for 1 hour at room temperature. Signals were visualized with the ECL Reagent (MilliporeSigma, 345818). Full, uncut gels are available in Supplemental Figure 7.

Gant61/JQ1 treatment. For in vitro treatment, Gant61 (MedChemExpress, HY-13901) and JQ1 (MedChemExpress, HY-13030) were dissolved in DMSO, and cells were treated using gradient concentrations. For in vivo treatment, JQ1 and Gant61 were dissolved and used according to the recommended guidelines $(48,49)$. Four-week-old Ctsk-CKO mice and littermate controls were injected with $50 \mathrm{mg} / \mathrm{kg}$ Gant61 or vehicle intraperitoneally every 2 days or $50 \mathrm{mg} / \mathrm{kg} \mathrm{JQ} 1$ or vehicle intraperitoneally daily. After 3 weeks, we performed radiographic assessment and histological analysis on these hind limbs.

Statistics. All results are presented as the mean \pm SEM. Comparisons between 2 groups were analyzed using 2-tailed, unpaired Student's $t$ test. ANOVA followed by Tukey's post hoc test was used when the data involved multiple-group comparisons.

Study approval. All experiments were performed according to the protocol approved by the Animal Care and Use Committee of the Institute of Biochemistry and Cell Biology, Shanghai Institutes for Biological Sciences, Chinese Academy of Sciences.

\section{Author contributions}

$\mathrm{HF}, \mathrm{WX}, \mathrm{YH}$, and WZ conceived the study and designed the experiments. HF, WX, and YH performed most of the research. JS, MK, and YY helped perform the research. BG, ZY, XC, YZ, and QB provided reagents and valuable comments and helped to analyze the data. HF, WX, YH, and WZ analyzed data and wrote the paper.

\section{Acknowledgments}

We thank S. Kato for Ctsk-Cre mice, Zunyi Zhang for Gli2 $2^{f / f l}$ mice, and Hongwei Ouyang for ScxGFP mice, and Zilong Qiu for Ai9 reporter mice. We also thank the cell biology core facility, the animal core facility, and the molecular biology core facility of Shanghai Institute of Biochemistry and Cell Biology for assistance. This work was supported in part by grants from the National Natural Science Foundation of China (NSFC) (81672769, 81725010, 81991512, and 81672119) and the Strategic Priority Research Program of the Chinese Academy of Sciences, grant XDB19000000. WZ is a scholar of the National Science Fund for Distinguished Young Scholars (NSFC) (no. 81725010).

Address correspondence to: Weiguo Zou, Institute of Biochemistry and Cell Biology, Shanghai Institutes for Biological Sciences, Chinese Academy of Sciences, 320 Yueyang Road, Building B 1403, Shanghai 200031, China. Phone: 86.21.54921320; Email: zouwg94@sibcb.ac.cn.
1. Xu R, Hu J, Zhou X, Yang Y. Heterotopic ossification: mechanistic insights and clinical challenges. Bone. 2018;109:134-142.

2. McCarthy EF, Sundaram M. Heterotopic ossification: a review. Skeletal Radiol. 2005;34(10):609-619.

3. Shehab D, Elgazzar AH, Collier BD. Heterotopic ossification. J Nucl Med. 2002;43(3):346-353.

4. Kaplan FS, Hahn GV, Zasloff MA. Heterotopic ossification: two rare forms and what they can teach us. JAm Acad Orthop Surg. 1994;2(5):288-296.

5. Agarwal S, et al. Scleraxis-lineage cells contribute to ectopic bone formation in muscle and tendon. Stem Cells. 2017;35(3):705-710.

6. Dey D, et al. Two tissue-resident progenitor lineages drive distinct phenotypes of heterotopic ossification. Sci Transl Med. 2016;8(366):366ra163.

7. Kan C, et al. Gli1-labeled adult mesenchymal stem/progenitor cells and hedgehog signaling contribute to endochondral heterotopic ossification. Bone. 2018;109:71-79.

8. Kan L, Peng CY, McGuire TL, Kessler JA. Glast-expressing progenitor cells contribute to heterotopic ossification. Bone. 2013;53(1):194-203.

9. Lees-Shepard JB, et al. Palovarotene reduces heterotopic ossification in juvenile FOP mice but exhibits pronounced skeletal toxicity. Elife. 2018;7:e40814.

10. Lees-Shepard JB, et al. Activin-dependent signaling in fibro/adipogenic progenitors causes fibrodysplasia ossificans progressiva. Nat Commun. 2018;9(1):471.

11. Olmsted-Davis EA, et al. Progenitors in peripheral nerves launch heterotopic ossification. Stem Cells Transl Med. 2017;6(4):1109-1119.

12. Suda RK, et al. Circulating osteogenic precursor cells in heterotopic bone formation. Stem Cells.
2009;27(9):2209-2219.

13. Bi Y, et al. Identification of tendon stem/progenitor cells and the role of the extracellular matrix in their niche. Nat Med. 2007;13(10):1219-1227.

14. Agarwal S, et al. Analysis of bone-cartilage-stromal progenitor populations in trauma induced and genetic models of heterotopic ossification. Stem Cells. 2016;34(6):1692-1701.

15. Bovée JV, Hogendoorn PC, Wunder JS, Alman BA. Cartilage tumours and bone development: molecular pathology and possible therapeutic targets. Nat Rev Cancer. 2010;10(7):481-488.

16. Deng Q, et al. Activation of hedgehog signaling in mesenchymal stem cells induces cartilage and bone tumor formation via Wnt/ $\beta$-catenin. Elife. 2019;8:e50208.

17. Karp SJ, Schipani E, St-Jacques B, Hunzelman J, Kronenberg H, McMahon AP. Indian hedgehog coordinates endochondral bone growth and mor- 
phogenesis via parathyroid hormone related-protein-dependent and -independent pathways. Development. 2000;127(3):543-548.

18. Mak KK, Chen MH, Day TF, Chuang PT, Yang Y. Wnt/beta-catenin signaling interacts differentially with Ihh signaling in controlling endochondral bone and synovial joint formation. Development. 2006;133(18):3695-3707.

19. Regard JB, et al. Activation of Hedgehog signaling by loss of GNAS causes heterotopic ossification. Nat Med. 2013;19(11):1505-1512.

20. Coquenlorge S, et al. GLI2 Modulated by SUFU and SPOP induces intestinal stem cell niche signals in development and tumorigenesis. Cell Rep. 2019;27(10):3006-3018.e4.

21. Hui CC, Angers S. Gli proteins in development and disease. Annu Rev Cell Dev Biol. 2011;27:513-537.

22. Debnath S, et al. Discovery of a periosteal stem cell mediating intramembranous bone formation. Nature. 2018;562(7725):133-139.

23. Han Y, et al. Lkb1 deletion in periosteal mesenchymal progenitors induces osteogenic tumors through mTORC1 activation. JClin Invest. 2019;129(5):1895-1909.

24. Nakamura T, et al. Estrogen prevents bone loss via estrogen receptor alpha and induction of Fas ligand in osteoclasts. Cell. 2007;130(5):811-823.

25. Yang $\mathrm{W}$, et al. Ptpn11 deletion in a novel progenitor causes metachondromatosis by inducing hedgehog signalling. Nature. 2013;499(7459):491-495.

26. Kusumbe AP, Ramasamy SK, Adams RH. Coupling of angiogenesis and osteogenesis by a specific vessel subtype in bone. Nature. 2014;507(7492):323-328.

27. Wang $X$, et al. Inhibition of overactive TGF- $\beta$ attenuates progression of heterotopic ossification in mice. Nat Commun. 2018;9(1):551.

28. Maeda T, et al. Conversion of mechanical force into TGF- $\beta$-mediated biochemical signals. Curr
Biol. 2011;21(11):933-941.

29. Asai S, et al. Tendon progenitor cells in injured tendons have strong chondrogenic potential: the CD105-negative subpopulation induces chondrogenic degeneration. Stem Cells. 2014;32(12):3266-3277.

30. Pryce BA, Watson SS, Murchison ND, Staverosky JA, Dünker N, Schweitzer R. Recruitment and maintenance of tendon progenitors by TGFbeta signaling are essential for tendon formation. Development. 2009;136(8):1351-1361.

31. Varjosalo M, Taipale J. Hedgehog: functions and mechanisms. Genes Dev. 2008;22(18):2454-2472.

32. Fu J, et al. GANT-61 inhibits pancreatic cancer stem cell growth in vitro and in NOD/SCID/ IL2R gamma null mice xenograft. Cancer Lett. 2013;330(1):22-32.

33. Lauth M, Bergström A, Shimokawa T, Toftgård R. Inhibition of GLI-mediated transcription and tumor cell growth by small-molecule antagonists. Proc Natl Acad Sci U S A. 2007;104(20):8455-8460.

34. Tang Y, et al. Epigenetic targeting of Hedgehog pathway transcriptional output through BET bromodomain inhibition. Nat Med. 2014;20(7):732-740.

35. Kan C, et al. BMP-dependent, injury-induced stem cell niche as a mechanism of heterotopic ossification. Stem Cell Res Ther. 2019;10(1):14.

36. Liu H, Xu J, Jiang R. Mkx-deficient mice exhibit Hedgehog signaling-dependent ectopic ossification in the Achilles tendons. J Bone Miner Res. 2019;34(3):557-569.

37. Hopyan S, et al. A mutant PTH/PTHrP type I receptor in enchondromatosis. Nat Genet. 2002;30(3):306-310.

38. Yin WC, et al. Dual regulatory functions of SUFU and targetome of GLI2 in SHH subgroup medulloblastoma. Dev Cell. 2019;48(2):167-183.e5.

39. Amakye D, Jagani Z, Dorsch M. Unraveling the therapeutic potential of the Hedgehog pathway in cancer. Nat Med. 2013;19(11):1410-1422.
40. Infante P, Alfonsi R, Botta B, Mori M, Di Marcotullio L. Targeting GLI factors to inhibit the Hedgehog pathway. Trends Pharmacol Sci. 2015;36(8):547-558.

41. Sugita D, et al. Indian hedgehog signaling promotes chondrocyte differentiation in enchondral ossification in human cervical ossification of the posterior longitudinal ligament. Spine. 2013;38(22):E1388-E1396.

42. Bai CB, Auerbach W, Lee JS, Stephen D, Joyner AL. Gli2, but not Gli1, is required for initial Shh signaling and ectopic activation of the Shh pathway. Development. 2002;129(20):4753-4761.

43. Hsu SH, Zhang X, Cheng S, Wunder JS, Hui CC, Alman BA. Suppressor of fused (Sufu) mediates the effect of parathyroid hormone-like hormone (Pthlh) on chondrocyte differentiation in the growth plate. JBiol Chem. 2012;287(43):36222-36228.

44. Corrales JD, Blaess S, Mahoney EM, Joyner AL. The level of sonic hedgehog signaling regulates the complexity of cerebellar foliation. Development. 2006;133(9):1811-1821.

45. Pryce BA, Brent AE, Murchison ND, Tabin CJ, Schweitzer R. Generation of transgenic tendon reporters, ScxGFP and ScxAP, using regulatory elements of the scleraxis gene. Dev Dyn. 2007;236(6):1677-1682.

46. Madisen L, et al. A robust and high-throughput Cre reporting and characterization system for the whole mouse brain. Nat Neurosci. 2010;13(1):133-140.

47. Niu N, et al. RNA-binding protein SAMD4 regulates skeleton development through translational inhibition of Mig6 expression. Cell Discov. 2017;3:16050.

48. Filippakopoulos P, et al. Selective inhibition of BET bromodomains. Nature. 2010;468(7327):1067-1073.

49. Neelakantan D, et al. EMT cells increase breast cancer metastasis via paracrine GLI activation in neighbouring tumour cells. Nat Commun. 2017;8:15773. 